\title{
Multivariate limit theorems in the context of long-range dependence
}

\author{
Shuyang Bai \\ Murad S. Taqqu
}

September 4, 2018

\begin{abstract}
We study the limit law of a vector made up of normalized sums of functions of long-range dependent stationary Gaussian series. Depending on the memory parameter of the Gaussian series and on the Hermite ranks of the functions, the resulting limit law may be (a) a multivariate Gaussian process involving dependent Brownian motion marginals, or (b) a multivariate process involving dependent Hermite processes as marginals, or (c) a combination. We treat cases (a), (b) in general and case (c) when the Hermite components involve ranks 1 and 2. We include a conjecture about case (c) when the Hermite ranks are arbitrary.
\end{abstract}

\section{Introduction}

A stationary time series displays long-range dependence if its auto-covariance decays slowly or if its spectral density diverges around the zero frequency. When there is long-range dependence, the asymptotic limits of various estimators are often either Brownian Motion or a Hermite process. The most common Hermite processes are fractional Brownian motion (Hermite process of order 1) and the Rosenblatt process (Hermite process of order 2), but there are Hermite processes of any order. Fractional Brownian motion is the only Gaussian Hermite process.

Most existing limit theorems involve univariate convergence, that is, convergence to a single limit process, for example, Brownian motion or a Hermite process ([3, 5, 24 $)$. In time series analysis, however, one often needs joint convergence, that is, convergence to a vector of processes. This is because one often needs to consider different statistics of the process jointly. See, for example, [11, 22]. We establish a number of results involving joint convergence, and conclude with a conjecture.

Our setup is as follows. Suppose $\left\{X_{n}\right\}$ is a stationary Gaussian series with mean 0, variance 1 and regularly varying auto-covariance

$$
\gamma(n)=L(n) n^{2 d-1}
$$

where $0<d<1 / 2$, and $L$ is a slowly varying function at infinity. This is often referred to "long-range dependence"(LRD) or "long memory" in the literature, and $d$ is called the memory parameter. The higher $d$, the stronger the dependence. The slow decay (1) of $\gamma(n)$ yields

$$
\sum_{n=-\infty}^{\infty}|\gamma(n)|=\infty
$$

Key words Long-range dependence; Gaussian process; Central limit theorems; Non-central limit theorems; Asymptotic independence; Multiple Wiener-Itô integrals

2010 AMS Classification: 60G18, 60F05 
The case where

$$
\sum_{n=-\infty}^{\infty}|\gamma(n)|<\infty
$$

is often referred to "short-range dependence" (SRD) or "short memory". See [1, 6, 8] for more details about these notions.

We are interested in the limit behavior of the finite-dimensional distributions (f.d.d.) of the following vector as $N \rightarrow \infty$ :

$$
\mathbf{V}_{N}(t)=\left(\frac{1}{A_{j}(N)} \sum_{n=1}^{[N t]}\left(G_{j}\left(X_{n}\right)-\mathbb{E} G_{j}\left(X_{n}\right)\right)\right)_{j=1, \ldots, J}
$$

where $G_{j}, j=1, \ldots, J$ are nonlinear functions, $t>0$ is the time variable, and $A_{j}(N)$ 's are appropriate normalizations which make the variance of each component at $t=1$ tend to 1 . Observe that the same sequence $\left\{X_{n}\right\}$ is involved in each component of $\mathbf{V}_{N}$, in contrast to [9] who consider the case $J=2$ and $\left\{\left(X_{n}, Y_{n}\right)\right\}$ is a bivariate Gaussian vector series.

Note also that convergence in f.d.d. implies that our results continue to hold if one replaces the single time variable $t$ in (2) with a vector $\left(t_{1}, \ldots, t_{J}\right)$ which would make $\mathbf{V}_{N}\left(t_{1}, \ldots, t_{J}\right)$ a random field.

Depending on the memory parameter of the Gaussian series and on the Hermite ranks of the functions (Hermite ranks are defined in Section 2), the resulting limit law for (2) may be:

(a) a multivariate Gaussian process with dependent Brownian motion marginals,

(b) or a multivariate process with dependent Hermite processes as marginals,

(c) or a combination.

We treat cases (a), (b) in general and case (c) when the Hermite components involve ranks 1 and 2 only. To address case (c), we apply a recent asymptotic independence theorem of Nourdin and Rosinski [16] of WienerItô integral vectors. We include a conjecture about case (c) when the Hermite ranks are arbitrary. We also prove that the Hermite processes in the limit are dependent on each other. Thus, in particular, fractional Brownian motion and the Rosenblatt process in the limit are dependent processes even though they are uncorrelated. Although our results are formulated in terms of convergence of f.d.d. , under some additional assumption, they extend to weak convergence in $D[0,1]^{J}(\mathrm{~J}$-dimensional product space where $D[0,1]$ is the space of Càdlàg functions on $[0,1]$ with the uniform metric), as noted in Theorem 3.11 at the end of Section 3.

The paper is structured as follows. We review the univariate results in Section 2 In Section 3 , we state the corresponding multivariate results. Section 4 contains the proofs of the theorems in Section 3 . Appendix $\mathrm{A}$ shows that the different representations of the Hermite processes are also equivalent in a multivariate setting. Appendix B refers to the results of [16] and concerns asymptotic independence of Wiener-Itô integral vectors.

\section{Review of the univariate results}

We review first results involving (2) when $J=1$ in (2). Assume that $G$ belongs to $L^{2}(\phi)$, the set of square-integrable functions with respect to the standard Gaussian measure $\phi$. This Hilbert space $L^{2}(\phi)$ has a complete orthogonal basis $\left\{H_{m}(x)\right\}_{m \geq 0}$, where $H_{m}$ is the Hermite polynomial defined as

$$
H_{m}(x)=(-1)^{m} \exp \left(\frac{x^{2}}{2}\right) \frac{d^{m}}{d x^{m}} \exp \left(\frac{-x^{2}}{2}\right),
$$

([14], Chapter 1.4). Therefore, every function $G \in L^{2}(\phi)$ admits the following type of expansion:

$$
G=\sum_{m \geq 0} g_{m} H_{m}
$$


where $g_{m}=(m !)^{-1} \int_{\mathbb{R}} G(x) H_{m}(x) d \phi(x)$.

Since $H_{0}(x)=1$ and since we always center the series $\left\{G\left(X_{n}\right)\right\}$ by subtracting its mean in (2), we may always assume $g_{0}=\mathbb{E} G\left(X_{n}\right)=0$. The smallest index $k \geq 1$ for which $g_{k} \neq 0$ in the expansion (3) is called the Hermite rank of $G$.

Since $\left\{X_{n}\right\}$ is a stationary Gaussian series, it has the following spectral representation

$$
X_{n}=\int_{\mathbb{R}} e^{i n x} d W(x)
$$

where $W$ is the complex Hermitian $(W(A)=\overline{W(-A)})$ Gaussian random measure specified by $\mathbb{E} W(A) \overline{W(B)}=$ $F(A \cap B)$. The measure $F$ is called the spectral distribution of $\left\{X_{n}\right\}$, is also called the control measure of $W$, and is defined by $\gamma(n)=\mathbb{E} X_{n} X_{0}=\int_{\mathbb{R}} e^{i n x} d F(x)$ (See [12], Chapter 3.2).

Multiple Wiener-Itô integrals ([13])

$$
I_{m}(K)=\int_{\mathbb{R}^{m}}^{\prime \prime} K\left(x_{1}, \ldots, x_{m}\right) d W\left(x_{1}\right) \ldots d W\left(x_{m}\right)
$$

where $\int_{\mathbb{R}^{m}}\left|K\left(x_{1}, \ldots, x_{m}\right)\right|^{2} d F\left(x_{1}\right) \ldots d F\left(x_{m}\right)<\infty$, play an important role because of the following connection between Hermite polynomials and multiple Wiener-Itô integrals ([14] Theorem 2.7.7):

$$
H_{m}\left(X_{n}\right)=\int_{\mathbb{R}^{m}}^{\prime \prime} e^{i n\left(x_{1}+\ldots+x_{m}\right)} d W\left(x_{1}\right) \ldots d W\left(x_{m}\right),
$$

where the double prime " indicates that one doesn't integrate on the hyper-diagonals $x_{j}= \pm x_{k}, j \neq k$. Throughout this paper, $I_{m}($.$) denotes a m$-tuple Wiener-Itô integral of the type in (5).

We now recall some well-known univariate results:

Theorem 2.1. (SRD Case.) Suppose the memory parameter d and the Hermite rank $k \geq 1$ of $G$ satisfy

$$
0<d<\frac{1}{2}\left(1-\frac{1}{k}\right)
$$

Then

$$
\frac{1}{A(N)} \sum_{n=1}^{[N t]} G\left(X_{n}\right) \stackrel{\text { f.d.d. }}{\longrightarrow} B(t),
$$

where $B(t)$ is a standard Brownian Motion, “ $\stackrel{f . d . d .}{\longrightarrow}$ " denotes convergence in finite-dimensional distributions along the time variable $t>0, A(N) \propto N^{1 / 2}$ is a normalization factor such that

$$
\lim _{N \rightarrow \infty} \operatorname{Var}\left(\frac{1}{A(N)} \sum_{n=1}^{N} G\left(X_{n}\right)\right)=1 .
$$

Remark 2.2. It can indeed be shown that in the setting of Theorem 2.1,

$$
\operatorname{Var}\left(\sum_{n=1}^{N} G\left(X_{n}\right)\right) \sim \sigma^{2} N,
$$

where

$$
\sigma^{2}=\sum_{m=k}^{\infty} g_{m}^{2} m ! \sum_{n=-\infty}^{\infty} \gamma(n)^{m} .
$$

Recall that the $g_{m}$ 's are the coefficients of the Hermite expansion of $G$, and $\gamma$ is the auto-covariance function of $\left\{X_{n}\right\}$. 
Remark 2.3. The condition $0<d<\frac{1}{2}\left(1-\frac{1}{k}\right)$ can be replaced with a weaker condition $\sum_{n=-\infty}^{\infty}|\gamma(n)|^{k}<\infty$ or equivalently $\sum_{n=-\infty}^{\infty}\left|\gamma_{G}(n)\right|<\infty$, where $\gamma_{G}(n)$ is the auto-covariance function of $\left\{G\left(X_{n}\right)\right\}$. See Theorem 4.6.1 in [8]. If $d=\frac{1}{2}\left(1-\frac{1}{k}\right)$ but as $N \rightarrow \infty, \sum_{n=-N}^{N}|\gamma(n)|^{k}=\sum_{-N}^{N} n^{-1}|L(n)|^{k}=: L^{*}(N) \rightarrow \infty$ is slowly varying, then one still gets convergence to Brownian motion (Theorem 1' of [3]), but with the normalization $A(N) \propto\left(N L^{*}(N)\right)^{1 / 2}$. For example, if the slowly varying function in (1) is $L(n) \sim c>0$, then $A(N) \propto$ $(N \ln N)^{1 / 2}$.

The original proof of Theorem 2.1 (3]) was done by a method of moments using the so-called diagram formulas ([18]), which provide explicit ways to compute the cumulants of Hermite polynomials of Gaussian random variable. Recently, a remarkable technique for establishing central limit theorems of multiple WienerItô integral was found by [17, 19, whereby in the multiple Wiener-Itô integral setting, convergence of the fourth moment, or some equivalent easier-to-check condition, implies directly the Gaussian limit. See Theorem 7.2.4 in [14] for a proof in the case $t=1$.

Theorem 2.4. (LRD Case.) Suppose that the memory parameter $d$ and the Hermite rank $k \geq 1$ of $G$ satisfy

$$
\frac{1}{2}\left(1-\frac{1}{k}\right)<d<\frac{1}{2}
$$

Then

$$
\frac{1}{A(N)} \sum_{n=1}^{[N t]} G\left(X_{n}\right) \stackrel{\text { f.d.d. }}{\longrightarrow} Z_{d}^{(k)}(t):=I_{k}\left(f_{k, d}^{(t)}\right),
$$

where the control measure of $I_{k}($.$) is Lebesgue, A(N) \propto N^{1+(d-1 / 2) k} L(N)^{k / 2}$ is a normalization such that

$$
\lim _{N \rightarrow \infty} \operatorname{Var}\left(\frac{1}{A(N)} \sum_{n=1}^{N} G\left(X_{n}\right)\right)=1,
$$

and

$$
f_{k, d}^{(t)}\left(x_{1}, \ldots, x_{k}\right)=b_{k, d} \frac{e^{i t\left(x_{1}+\ldots+x_{k}\right)}-1}{i\left(x_{1}+\ldots+x_{k}\right)}\left|x_{1}\right|^{-d} \ldots\left|x_{k}\right|^{-d}
$$

where

$$
b_{k, d}=\left(\frac{(k(d-1 / 2)+1)(2 k(d-1 / 2)+1)}{k !(2 \Gamma(1-2 d) \sin (d \pi))^{k}}\right)^{1 / 2}
$$

is the normalization constant to guarantee unit variance for $Z^{(k)}(1)$.

For a proof, see [5] and [21]. The process $Z_{d}^{(k)}(t)$ appearing in the limit is called a Hermite process.

Remark 2.5. It can indeed be shown that in the setting of Theorem 2.4,

$$
\operatorname{Var}\left(\sum_{n=1}^{N} G\left(X_{n}\right)\right)=L_{G}(N) N^{2 d_{G}+1}
$$

for some slowly varying function $L_{G}(N) \propto L(N)^{k}$ and $d_{G}=(d-1 / 2) k+1 / 2$ (see e.g. (3.3.8) in [8]). Since $d<1 / 2$, increasing the Hermite rank $k$ decreases the memory parameter $d_{G}$, hence decreases the dependence. Note that if $k \geq 2$, then the variance growth of $\left\{G\left(X_{n}\right)\right\}$ in (9) is slower than the variance growth of $\left\{X_{n}\right\}$, $\operatorname{Var}\left(\sum_{n=1}^{N} X_{n}\right)=L_{0}(N) N^{2 d+1}$ for some slowly varying function $L_{0}$, but is always faster than the variance growth $\sigma^{2} N$ in the SRD case in (7).

The process $Z_{d}^{(1)}(t), t \geq 0$ is a Gaussian process called fractional Brownian motion, and $Z_{d}^{(2)}(t), t \geq 0$ is a non-Gaussian process called Rosenblatt process. The Hermite processes $Z_{d}^{(k)}(t)$ are all so-called self-similar processes ([7]). 


\section{Multivariate convergence results}

Our aim is to study the limit of (2), and in particular, to extend Theorem 2.1 (SRD) and Theorem 2.4 (LRD) to a multivariate setting.

Suppose that for each $j=1, \ldots, J$, the function the functions $G_{j}$ in (2) belongs to $L^{2}(\phi)$, has Hermite rank $k_{j}$ and admits Hermite expansion $\sum_{m=k_{j}}^{\infty} g_{m, j} H_{m}$ (see (3)).

We start with the pure SRD case where every component $\left\{G_{j}\left(X_{n}\right)\right\}$ of $\mathbf{V}_{N}(t)$ in (2) is SRD.

Theorem 3.1. (Pure SRD Case.) If the memory parameter $d$ is small enough so that all $\left\{G_{j}\left(X_{n}\right)\right\}, j=$ $1, \ldots, J$ are $S R D$, that is,

$$
d<\frac{1}{2}\left(1-\frac{1}{k_{j}}\right), j=1, \ldots, J,
$$

then in (2)

$$
\mathbf{V}_{N}(t) \stackrel{\text { f.d.d. }}{\longrightarrow} \mathbf{B}(t)
$$

as $N \rightarrow \infty$, where the normalization $A_{j}(N) \propto N^{1 / 2}$ is such that for $j=1, \ldots, J$,

$$
\lim _{N \rightarrow \infty} \operatorname{Var}\left(\frac{1}{A_{j}(N)} \sum_{n=1}^{N} G_{j}\left(X_{n}\right)\right)=1 .
$$

Here $\mathbf{B}(t)=\left(B_{1}(t), \ldots, B_{J}(t)\right)$ is a multivariate Gaussian process with standard Brownian motions as marginals, and where the cross-covariance between two components is

$$
\begin{aligned}
\operatorname{Cov}\left(B_{j_{1}}\left(t_{1}\right), B_{j_{2}}\left(t_{2}\right)\right) & =\lim _{N \rightarrow \infty} \operatorname{Cov}\left(V_{N, j_{1}}\left(t_{1}\right), V_{N, j_{2}}\left(t_{2}\right)\right) \\
& =\left(t_{1} \wedge t_{2}\right)\left[\frac{1}{\sigma_{j_{1} \sigma_{j_{2}}}} \sum_{m=k_{j_{1}} \vee k_{j_{2}}}^{\infty} g_{m, j_{1}} g_{m, j_{2}} m ! \sum_{n=-\infty}^{\infty} \gamma(n)^{m}\right]
\end{aligned}
$$

where

$$
\sigma_{j}^{2}=\sum_{m=k_{j}}^{\infty} g_{m, j}^{2} m ! \sum_{n=-\infty}^{\infty} \gamma(n)^{m} .
$$

This theorem is proved in Section 4.1

Example 3.2. Assume that the auto-covariance function $\gamma(n) \sim n^{2 d-1}$ as $n \rightarrow \infty$. Let $J=2, G_{1}(x)=$ $a H_{2}(x)+b H_{3}(x)=b x^{3}+a x^{2}-3 b x-a, G_{2}(x)=c H_{3}(x)=c x^{3}-3 c x$ and $0<d<1 / 4$. Then in (12), $\sigma_{1}^{2}=2 a^{2} \sum_{n=-\infty}^{\infty} \gamma(n)^{2}+6 b^{2} \sum_{n=-\infty}^{\infty} \gamma(n)^{3}, \sigma_{2}^{2}=6 c^{2} \sum_{n=-\infty}^{\infty} \gamma(n)^{3}$, and

$$
\left(\frac{1}{N^{1 / 2}} \sum_{n=1}^{[N t]}\left(X_{n}^{2}-1\right), \frac{1}{N^{1 / 2}} \sum_{n=1}^{[N t]}\left(X_{n}^{3}-3 X_{n}\right)\right) \stackrel{\text { f.d.d. }}{\longrightarrow}\left(\sigma_{1} B_{1}(t), \sigma_{2} B_{2}(t)\right),
$$

where the Brownian motions $B_{1}$ and $B_{2}$ have the covariance structure:

$$
\operatorname{Cov}\left(B_{1}\left(t_{1}\right), B_{2}\left(t_{2}\right)\right)=6 b \frac{t_{1} \wedge t_{2}}{\sigma_{1} \sigma_{2}} \sum_{n=-\infty}^{\infty} \gamma(n)^{3} .
$$

$B_{1}$ and $B_{2}$ are independent when $b=0$.

Next we consider the case where every component $\left\{G_{j}\left(X_{n}\right)\right\}$ of $\mathbf{V}_{N}(t)$ in (2) is LRD.

Theorem 3.3. (Pure LRD Case.) If the memory parameter $d$ is large enough so that all $G_{j}\left(X_{n}\right), j=$ $1, \ldots, J$ are $L R D$, that is,

$$
d>\frac{1}{2}\left(1-\frac{1}{k_{j}}\right), j=1, \ldots, J
$$


then in (2),

$$
\mathbf{V}_{N}(t) \stackrel{\text { f.d.d. }}{\longrightarrow} \mathbf{Z}_{d}^{\mathbf{k}}(t):=\left(I_{k_{1}}\left(f_{k_{1}, d}^{(t)}\right), \ldots, I_{k_{J}}\left(f_{k_{J}, d}^{(t)}\right)\right)
$$

where the normalization $A_{j}(N) \propto N^{1+(d-1 / 2) k_{j}} L(N)^{k_{j} / 2}$ is such that for $j=1, \ldots, J$,

$$
\lim _{N \rightarrow \infty} \operatorname{Var}\left(\frac{1}{A_{j}(N)} \sum_{n=1}^{N} G_{j}\left(X_{n}\right)\right)=1 .
$$

Each component of $\mathbf{Z}_{d}^{\mathbf{k}}(t):=\left(Z_{d}^{\left(k_{1}\right)}(t), \ldots, Z_{d}^{\left(k_{J}\right)}(t)\right)$ is a standard Hermite process, and $I_{k}($.$) denotes k$ tuple Wiener-Itô integral with respect to a common complex Hermitian Gaussian random measure $W$ with Lebesgue control measure, and

$$
f_{k, d}^{(t)}\left(x_{1}, \ldots, x_{k}\right)=b_{k, d} \frac{e^{i t\left(x_{1}+\ldots+x_{k}\right)}-1}{i\left(x_{1}+\ldots+x_{k}\right)}\left|x_{1}\right|^{-d} \ldots\left|x_{k}\right|^{-d},
$$

where $b_{k, d}$ 's are the same normalization constants as in Theorem 2.4.

This theorem is proved in Section 4.2

Example 3.4. Assume that auto-covariance function $\gamma(n) \sim n^{2 d-1}$ as $n \rightarrow \infty$. Let $J=2, G_{1}(x)=H_{1}(x)=$ $x, G_{2}(x)=H_{2}(x)=x^{2}-1,1 / 4<d<1 / 2$, then

$$
\left(\frac{1}{N^{1 / 2+d}} \sum_{n=1}^{[N t]} X_{n}, \frac{1}{N^{2 d}} \sum_{n=1}^{[N t]}\left(X_{n}^{2}-1\right)\right) \stackrel{\text { f.d.d. }}{\longrightarrow}\left(\frac{1}{d(2 d+1)} Z_{d}^{(1)}(t), \frac{1}{d(4 d-1)} Z_{d}^{(2)}(t)\right),
$$

where the standard fractional Brownian motion $Z_{d}^{(1)}(t)$ and standard Rosenblatt process $Z_{d}^{(2)}(t)$ share the same random measure in the Wiener-Itô integral representation. The components $Z_{d}^{(1)}$ and $Z_{d}^{(2)}$ are uncorrelated but dependent as stated below.

In Theorem 3.3, the marginal Hermite processes $Z_{d}^{\left(k_{1}\right)}(t)=I_{k_{1}}\left(f_{k_{1}, d}^{(t)}\right), \ldots, Z_{d}^{\left(k_{J}\right)}(t)=I_{k_{J}}\left(f_{k_{J}, d}^{(t)}\right)$ are dependent on each other. To prove this, we use a different representation of the Hermite process, namely, the positive half-axis representation given in (43).

Proposition 3.5. The marginal Hermite processes $Z_{d}^{\left(k_{1}\right)}, \ldots, Z_{d}^{\left(k_{J}\right)}$ involved in Theorem 3.3 are dependent.

Proof. From [25], we have the following criterion for the independence of multiple Wiener-Itô integrals: suppose that symmetric $g_{1} \in L^{2}\left(\mathbb{R}_{+}^{p}\right)$ and $g_{2} \in L^{2}\left(\mathbb{R}_{+}^{q}\right)$. Then $I_{p}\left(g_{1}\right)$ and $I_{q}\left(g_{2}\right)(p, q \geq 1)$ are independent if and only if

$$
g_{1} \otimes_{1} g_{2}:=\int_{\mathbb{R}_{+}} g_{1}\left(x_{1}, \ldots, x_{p-1}, u\right) g_{2}\left(x_{p}, \ldots, x_{p+q-2}, u\right) d u=0 \text { in } L^{2}\left(\mathbb{R}_{+}^{p+q-2}\right) .
$$

We shall apply this criterion to the positive half-axis integral representation (43) of Hermite processes (see also [21]):

$$
Z_{d}^{(k)}(t)=c_{k, d} I_{k}\left(g_{k, d}^{(t)}\left(x_{1}, \ldots, x_{k}\right)\right):=c_{k, d} \int_{\mathbb{R}_{+}^{k}}^{\prime}\left[\int_{0}^{t} \prod_{j=1}^{k} x_{j}^{-d}\left(1-s x_{j}\right)_{+}^{d-1} d s\right] d B\left(x_{1}\right) \ldots d B\left(x_{k}\right),
$$

where $B$ is Brownian motion, the prime ' indicates the exclusion of diagonal $x_{j}=x_{k}, j \neq k$ and $c_{k, d}$ is some normalization constant. In fact, for a vector made up of Hermite processes sharing the same random measure in their Wiener-Itô integral representation, the joint distribution does not change when switching from one representation of Hermite process to another. See Appendix $\mathrm{A}$. 
One can then see (let $t=1$ and thus $g_{k, d}:=g_{k, d}^{(1)}$ for simplicity) that for all $\left(x_{1}, \ldots, x_{p+q-2}\right) \in \mathbb{R}_{+}^{p+q-2}$ :

$$
\begin{aligned}
& \left(g_{p, d} \otimes_{1} g_{q, d}\right)\left(x_{1}, \ldots, x_{p+q-2}\right) \\
= & \int_{\mathbb{R}_{+}}\left(\int_{0}^{1} \prod_{j=1}^{p-1} x_{j}^{-d}\left(1-s x_{j}\right)_{+}^{d-1} u^{-d}(1-s u)_{+}^{d-1} d s \int_{0}^{1} \prod_{j=p}^{p+q-2} x_{j}^{-d}\left(1-s x_{j}\right)_{+}^{d-1} u^{-d}(1-s u)_{+}^{d-1} d s\right) d u>0
\end{aligned}
$$

because every term involved in the integrand is positive.

Theorem 3.1 and Theorem 3.3 describe the convergence of $\mathbf{V}_{N}(t)$ in (2) when the $\left\{G_{j}\left(X_{n}\right)\right\}, j=1, \ldots, J$ are all purely SRD or purely LRD. However, when the components in $\mathbf{V}_{N}(t)$ are mixed, that is, some of them are SRD and some of them are LRD, it is not immediately clear what the limit behavior is and also what the inter-dependence structure between the SRD and LRD limit components is. We show that the SRD part and LRD part are asymptotically independent so that one could join the limits of Theorem 3.1 and Theorem 3.3 together, in the case when the $G_{j}$ 's in the LRD part only involve the 2 lowest Hermite ranks, namely, $k=1$ or $k=2$. This is stated in the next theorem where the letter "S" refers to the SRD part and "L" to the LRD part.

Theorem 3.6. (Special SRD and LRD Mixed Case.) Separate the SRD and LRD parts of $\mathbf{V}_{N}(t)$ in (2), that is, let $\mathbf{V}_{N}(t)=\left(\mathbf{S}_{N}(t), \mathbf{L}_{N}(t)\right)$, where

$$
\begin{aligned}
& \mathbf{S}_{N}(t)=\left(\frac{1}{A_{1, S}(N)} \sum_{n=1}^{[N t]} G_{1, S}\left(X_{n}\right), \ldots, \frac{1}{A_{J_{S}, S}(N)} \sum_{n=1}^{[N t]} G_{J_{S}, S}\left(X_{n}\right)\right) \\
& \mathbf{L}_{N}(t)=\left(\frac{1}{A_{1, L}(N)} \sum_{n=1}^{[N t]} G_{1, L}\left(X_{n}\right), \ldots, \frac{1}{A_{J_{L}, L}(N)} \sum_{n=1}^{[N t]} G_{J_{L}, L}\left(X_{n}\right)\right),
\end{aligned}
$$

where $G_{j, S}$ has Hermite rank $k_{j, S}$, and $G_{j, L}$ has Hermite rank $k_{j, L}, A_{j, S} \propto N^{1 / 2}$ and $A_{j, L} \propto N^{1+(d-1 / 2) k_{j, L}} L(N)^{k_{j, L} / 2}$ are the correct normalization factors such that for $j=1, \ldots, J_{S}$ and $j=1, \ldots, J_{L}$ respectively,

$$
\lim _{N \rightarrow \infty} \operatorname{Var}\left(\frac{1}{A_{j, S}(N)} \sum_{n=1}^{N} G_{j, S}\left(X_{n}\right)\right)=1, \quad \lim _{N \rightarrow \infty} \operatorname{Var}\left(\frac{1}{A_{j, L}(N)} \sum_{n=1}^{N} G_{j, L}\left(X_{n}\right)\right)=1 .
$$

In addition,

$$
\frac{1}{2}\left(1-\frac{1}{k_{j_{L}, L}}\right)<d<\frac{1}{2}\left(1-\frac{1}{k_{j_{S}, S}}\right) \quad \text { for all } j_{S}=1, \ldots, J_{S}, j_{L}=1, \ldots, J_{L},
$$

where we allow arbitrary values for $k_{j, S}$ but only $k_{j, L}=1$ or 2 . (Condition (19) makes all $\left\{G_{j, S}\left(X_{n}\right)\right\}$ SRD and all $\left.\left\{G_{j, L}\left(X_{n}\right)\right\} L R D.\right)$

Then we have

$$
\left(\mathbf{S}_{N}(t), \mathbf{L}_{N}(t)\right) \stackrel{\text { f.d.d. }}{\longrightarrow}\left(\mathbf{B}(t), \mathbf{Z}_{d}^{\left(\mathbf{k}_{L}\right)}(t)\right)
$$

where the multivariate Gaussian process $\mathbf{B}(t)$ is given in (3.1) and the multivariate standard Hermite process $\mathbf{Z}_{d}^{\left(\mathbf{k}_{L}\right)}(t)$ is given in (3.3). Moreover, the vectors $\mathbf{B}(t)$ and $\mathbf{Z}_{d}^{\left(\mathbf{k}_{L}\right)}(t)$ are independent.

This theorem is proved in Section 4.3. Observe that while $\mathbf{B}(t)$ is made up of correlated Brownian motions, it follows from Theorem 3.6 that if $\mathbf{Z}_{d}^{(k)}(t)$ contains fractional Brownian motion as a component, then the fractional Brownian motion will be independent of any Brownian motion component of $\mathbf{B}(t)$.

We conjecture the following:

Conjecture 3.7. Theorem 3.0 holds without the restriction that $k_{j, L}$ be 1 or 2 . 
Example 3.8. Assume that the auto-covariance function $\gamma(n) \sim n^{2 d-1}$ as $n \rightarrow \infty$. Let $J=2, G_{1}(x)=$ $H_{2}(x)=x^{2}-1, G_{2}(x)=H_{3}(x)=x^{3}-3 x, 1 / 4<d<1 / 3$, then $\sigma^{2}=6 \sum_{n=-\infty}^{\infty} \gamma(n)^{3}$ and

$$
\left(\frac{1}{N^{2 d}} \sum_{n=1}^{[N t]}\left(X_{n}^{2}-1\right), \frac{1}{N^{1 / 2}} \sum_{n=1}^{[N t]}\left(X_{n}^{3}-3 X_{n}\right)\right) \stackrel{\text { f.d.d. }}{\longrightarrow}\left(\frac{1}{d(4 d-1)} Z_{d}^{(2)}(t), \sigma B(t)\right) \text {. }
$$

where the standard Rosenblatt process $Z_{d}^{(2)}(t)$ and the standard Brownian motion $B(t)$ are independent.

The proof of Theorem 3.6 is based a recent result in [16] which characterizes the asymptotic momentindependence of series of multiple Wiener-Itô integral vectors. We also note that in Proposition 5.3 (2) of [16], a special case of Theorem 3.6 with $J_{S}=J_{L}=1$ and LRD part involving Hermite rank $k_{1, L}=2$ is treated. To go from moment-independence to independence, however, requires moment-determinancy of the limit, which we know holds when the Hermite rank $k=1,2$, that is, in the Gaussian and Rosenblatt cases. If some other Hermite distribution (marginal distribution of Hermite process) $Z_{d}^{(k)}(k \geq 3)$ is momentdeterminate, then we will allow $k_{j, L}=k$ in Theorem 3.6. So to this end, the moment-problem of general Hermite distributions is of great interest.

Remark 3.9. As mentioned in Remark 2.3, the border case $d_{j}=\frac{1}{2}\left(1-\frac{1}{k_{j}}\right)$ often leads to convergence to Brownian motion as well. In fact, Theorem 3.1 and Theorem 3.6 continue to hold if we extend the definition of SRD to the case whenever the limit is Brownian motion regardless of the normalization.

In Theorem 3.1, Theorem 3.3 and Theorem 3.6 we stated the results only in terms of convergence in finite-dimensional distributions, but in fact they hold under weak convergence in $D[0,1]^{J}$ (J-dimensional product space where $D[0,1]$ is the space of Càdlàg functions on $[0,1]$ with the uniform metric). If one can check that every component of $\mathbf{V}_{N}(t)$ is tight, then the vector $\mathbf{V}_{N}(t)$ is tight:

Lemma 3.10. Univariate tightness in $D[0,1]$ implies multivariate tightness in $D[0,1]^{J}$.

Proof. Suppose every component $X_{j, N}$ (a random element in $S=D[0,1]$ with uniform metric $d$ ) of the J-dimensional random element $\mathbf{X}_{N}$ is tight, that is, given any $\epsilon>0$, there exists a compact set $K_{j}$ in $D[0,1]$, so that for all $N$ large enough:

$$
P\left(X_{j, N} \in K^{c}\right)<\epsilon
$$

where $K_{j}^{c}$ denotes the complement of $K_{j}$. If $K=K_{1} \times \ldots \times K_{J}$, then $K$ is compact in the product space $S^{J}$. We can associate $S^{J}$ with any compatible metric, e.g., for $\mathbf{X}, \mathbf{Y} \in S^{J}$,

$$
d_{m}(\mathbf{X}, \mathbf{Y}):=\max _{1 \leq j \leq J}\left(d\left(X_{1}, Y_{1}\right), \ldots, d\left(X_{J}, Y_{J}\right)\right) .
$$

The sequence $\mathbf{X}_{N}$ is tight on $D[0,1]^{J}$ since

$$
P\left(\mathbf{X}_{N} \in K^{c}\right)=P\left(\cup_{j=1}^{J}\left\{X_{j, N} \in K_{j}^{c}\right\}\right) \leq \sum_{j=1}^{J} P\left(X_{j, N} \in K_{j}^{c}\right)<J \epsilon .
$$

The univariate tightness is shown in 24 for the LRD case. The tightness for the SRD case was considered in 4 p. 328 and holds under the following additional assumption, that $\left\{G\left(X_{n}\right)\right\}$ is SRD, with

$$
\sum_{k=1}^{\infty} 3^{k / 2}(k !)^{1 / 2}\left|g_{k}\right|<\infty
$$

where $g_{k}$ is the $k$-th coefficient of Hermite expansion (3) of $G$. Observe that (21) is a strengthening of the basic condition: $\mathbb{E}\left[G\left(X_{0}\right)^{2}\right]=\sum_{k=1} k ! g_{k}^{2}<\infty$. Hence we have:

Theorem 3.11. Suppose that condition (21) holds for the short-range dependent components. Then the convergence in Theorem 3.1, Theorem 3.3 and Theorem 3.6 holds as weak convergence in $D[0,1]^{J}$.

Condition (21) is satisfied in the important special case where $G$ is a polynomial of finite order. 


\section{Proofs of the multivariate convergence results}

\subsection{Proof of Theorem 3.1}

We start with a number of lemmas. The first yields the limit covariance structure in (11).

Lemma 4.1. Assume that $\sum_{n}|\gamma(n)|^{m}<\infty$, then as $N \rightarrow \infty$ :

$$
\frac{1}{N} \sum_{n_{1}=1}^{\left[N t_{1}\right]} \sum_{n_{2}=1}^{\left[N t_{2}\right]} \gamma\left(n_{1}-n_{2}\right)^{m} \rightarrow\left(t_{1} \wedge t_{2}\right) \sum_{n=-\infty}^{\infty} \gamma(n)^{m}
$$

Proof. Denote the left-hand side of (22) by $S_{N}$. Let $a=t_{1} \wedge t_{2}$, and $b=t_{1} \vee t_{2}$, and

$$
S_{N, 1}=\frac{1}{N} \sum_{n_{1}=1}^{[N a]} \sum_{n_{2}=1}^{[N a]} \gamma\left(n_{1}-n_{2}\right)^{m}, \quad S_{N, 2}=\frac{1}{N} \sum_{n_{1}=1}^{[N a]} \sum_{n_{2}=[N a]+1}^{[N b]} \gamma\left(n_{1}-n_{2}\right)^{m}
$$

so $S_{N}=S_{N, 1}+S_{N, 2}$. We have as $N \rightarrow \infty$,

$$
S_{N, 1}=a \sum_{n_{1}=-[N a]+1}^{[N a]-1} \frac{[N a]-|n|}{N a} \gamma(n)^{m} \rightarrow a \sum_{n=-\infty}^{\infty} \gamma(n)^{m} .
$$

We hence need to show that $S_{N, 2} \rightarrow 0$. Let $c(n)=\gamma(n)^{m}$, then

$$
S_{N, 2} \leq \frac{1}{N} \sum_{n_{1}=1}^{[N a]} \sum_{n_{2}=[N a]+1}^{[N b]}\left|c\left(n_{2}-n_{1}\right)\right|=\frac{1}{N} \sum_{n_{1}=1}^{[N a]} c_{N, n_{1}}=\int_{0}^{a} f_{N}(u) d u,
$$

where

$$
c_{N, n_{1}}:=\sum_{n_{2}=[N a]+1}^{[N b]}\left|c\left(n_{2}-n_{1}\right)\right|=\sum_{n_{2}=1}^{[N b]-[N a]}\left|c\left([N a]+n_{2}-n_{1}\right)\right|
$$

and for $u \in(0, a)$,

$$
\begin{aligned}
f_{N}(u): & =\sum_{n_{1}=1}^{[N a]} c_{N, n_{1}} \mathbf{1}_{\left[\frac{n_{1}-1}{N}, \frac{n_{1}}{N}\right)}(u) \\
& =\sum_{n_{2}=1}^{[N b]-[N a]} \sum_{n_{1}=1}^{[N a]}\left|c\left([N a]+n_{2}-n_{1}\right)\right| \mathbf{1}_{\left[\frac{n_{1}-1}{N}, \frac{n_{1}}{N}\right)}(u) \\
& =\sum_{n_{2}=1}^{[N b-N a]}\left|c\left([N a]-[N u]-1+n_{2}\right)\right| .
\end{aligned}
$$

Now observe that $f_{N}(u) \leq \sum_{n=-\infty}^{\infty}|c(n)|=\sum_{n=-\infty}^{\infty}|\gamma(n)|^{m}<\infty$ and that $[N a]-[N u] \rightarrow \infty$ as $N \rightarrow \infty$. Applying the Dominated Convergence Theorem, we deduce $f_{N}(u) \rightarrow 0$ on $(0, a)$. Applying the Dominated Convergence Theorem again, we conclude that $S_{N, 2} \rightarrow 0$.

Now we introduce some notations, setting for $G \in L^{2}(\phi)$,

$$
S_{N, t}(G):=\frac{1}{\sqrt{N}} \sum_{n=1}^{[N t]} G\left(X_{n}\right)
$$


The Hermite expansion of each $G_{j}$ is

$$
G_{j}=\sum_{m=k_{j}}^{\infty} g_{m, j} H_{m}
$$

if $G_{j}$ has Hermite rank $k_{j}$. Since we are in the pure SRD case, we have as in Remark 2.3, that the autocovariance function $\gamma(n)$ of $\left\{X_{n}\right\}$

$$
\sum_{n=-\infty}^{\infty}|\gamma(n)|^{k_{j}}<\infty, \quad \text { for } j=1, \ldots, J
$$

The following lemma states that it suffices to replace a general $G_{j}$ with a finite linear combination of Hermite polynomials:

Lemma 4.2. If Theorem 3.1 holds with a finite linear combination of Hermite polynomials $G_{j}=\sum_{m=k_{j}}^{M} a_{m, j} H_{m}$ for any $M \geq \max _{j}\left(k_{j}\right)$ and any $a_{m, j}$, then it also holds for any $G_{j} \in L^{2}(\phi)$.

Proof. First we obtain an $L^{2}$ bound for $S_{N, t}\left(H_{m}\right)$. By $\mathbb{E} H_{m}(X) H_{m}(Y)=m ! \mathbb{E}(X Y)^{m}$ (Proposition 2.2.1 in [14]), for $m \geq 1$,

$$
\begin{aligned}
\mathbb{E}\left(S_{N, t}\left(H_{m}\right)\right)^{2} & =\frac{1}{N} \sum_{n_{1}, n_{2}=1}^{[N t]} \mathbb{E} H_{m}\left(X_{n_{1}}\right) H_{m}\left(X_{n_{2}}\right)=\frac{m !}{N} \sum_{n_{1}, n_{2}=1}^{[N t]} \gamma\left(n_{1}-n_{2}\right)^{m} \\
& =t m ! \sum_{n=1-[N t]}^{[N t]-1} \frac{[N t]-|n|}{N t} \gamma(n)^{m} \leq t m ! \sum_{n=-\infty}^{\infty}|\gamma(n)|^{m} .
\end{aligned}
$$

Next, fix any $\epsilon>0$. By (25) and $\|G\|_{L^{2}(\phi)}^{2}=\sum_{m=0}^{\infty} g_{m}^{2} m$ !, for $M$ large enough, one has

$$
\begin{aligned}
& \mathbb{E}\left|S_{N, t}\left(G_{j}\right)-S_{N, t}\left(\sum_{m=k_{j}}^{M} g_{m, j} H_{m}\right)\right|^{2}=\mathbb{E}\left|S_{N, t}\left(\sum_{m=M+1}^{\infty} g_{m, j} H_{m}\right)\right|^{2} \\
= & \sum_{m=M+1}^{\infty} g_{m, j}^{2} \mathbb{E}\left(S_{N, t}\left(H_{m}\right)\right)^{2} \leq t \sum_{n=-\infty}^{\infty}|\gamma(n)|^{k_{j}} \sum_{m=M+1}^{\infty} g_{m, j}^{2} m ! \leq \epsilon t .
\end{aligned}
$$

Therefore, the $J$-vector

$$
\mathbf{V}_{N, M}(t)=\left(S_{N, t}\left(\sum_{m=k_{1}}^{M} g_{m, 1} H_{m}\right), \ldots, S_{N, t}\left(\sum_{m=k_{J}}^{M} g_{m, j} H_{m}\right)\right)
$$

satisfies $\lim \sup _{N} \mathbb{E}|| \mathbf{V}_{N, M}(t)-\left.\mathbf{V}_{N}(t)\right|^{2} \leq J \epsilon t$, and thus

$$
\lim _{M} \limsup _{N} \mathbb{E}\left|\mathbf{V}_{N, M}(t)-\mathbf{V}_{N}(t)\right|^{2}=0 .
$$

By assumption, we have as $N \rightarrow \infty \mathbf{V}_{N, M}(t) \stackrel{\text { f.d.d. }}{\longrightarrow} \mathbf{B}_{M}(t)=\left(B_{M, 1}, \ldots, B_{M, J}\right)$, where the multivariate Gaussian $\mathbf{B}_{M}(t)$ has (scaled) Brownian motions as marginals with a covariance structure computed using 
Lemma 4.1 as follows:

$$
\begin{aligned}
\mathbb{E}\left(B_{M, j_{1}}\left(t_{1}\right) B_{M, j_{2}}\left(t_{2}\right)\right) & =\lim _{N \rightarrow \infty} \mathbb{E}\left(S_{N, t_{1}}\left(\sum_{m=k_{j_{1}}}^{M} g_{m, j_{1}} H_{m}\right) S_{N, t_{2}}\left(\sum_{m=k_{j_{2}}}^{M} g_{m, j_{2}} H_{m}\right)\right) \\
& =\lim _{N \rightarrow \infty} \sum_{m=k_{j_{1}} \vee k_{j_{2}}}^{M} g_{m, j_{1}} g_{m, j_{2}} m ! \sum_{n_{1}=1}^{\left[N t_{1}\right]\left[N t_{2}\right]} \gamma\left(n_{1}-n_{2}\right)^{m}=1 \\
& =\left(t_{1} \wedge t_{2}\right) \sum_{m=k_{j_{1}} \vee k_{j_{2}}}^{M} g_{m, j_{1}} g_{m, j_{2}} m ! \sum_{n=-\infty}^{\infty} \gamma(n)^{m} .
\end{aligned}
$$

Furthermore, as $M \rightarrow \infty, \mathbf{B}_{M}(t)$ tends in f.d.d. to $\mathbf{B}(t)$, which is a multivariate Gaussian process with the following covariance structure:

$$
\mathbb{E}\left(B_{j_{1}}\left(t_{1}\right) B_{j_{2}}\left(t_{2}\right)\right)=\left(t_{1} \wedge t_{2}\right) \sum_{m=k_{j_{1}} \vee k_{j_{2}}}^{\infty} g_{m, j_{1}} g_{m, j_{2}} m ! \sum_{n=-\infty}^{\infty} \gamma(n)^{m} .
$$

Therefore, applying the triangular argument in [2] Theorem 3.2, we have

$$
\mathbf{V}_{N}(t) \stackrel{\text { f.d.d. }}{\longrightarrow} \mathbf{B}(t)
$$

The proof of Theorem 3.1 about the pure SRD case relies on [14 Theorem 6.2.3, which says that for multiple Wiener-Itô integrals, univariate convergence to normal random variables implies joint convergence to a multivariate normal. We state it as follows:

Lemma 4.3. Let $J \geq 2$ and $k_{1}, \ldots, k_{j}$ be some fixed positive integers. Consider vectors

$$
\mathbf{V}_{N}=\left(V_{N, 1}, \ldots, V_{N, J}\right):=\left(I_{k_{1}}\left(f_{N, 1}\right), \ldots, I_{k_{J}}\left(f_{N, J}\right)\right)
$$

with $f_{N, j}$ in $L^{2}\left(\mathbb{R}^{k_{j}}\right)$. Let $C$ be a symmetric non-negative definite matrix such that

$$
\mathbb{E}\left(V_{N, i} V_{N, j}\right) \rightarrow C(i, j) .
$$

Then the univariate convergence as $N \rightarrow \infty$

$$
V_{N, j} \stackrel{d}{\rightarrow} N(0, C(j, j)) \quad j=1, \ldots, J
$$

implies the joint convergence

$$
\mathbf{V}_{N} \stackrel{d}{\rightarrow} N(\mathbf{0}, C)
$$

We now prove Theorem 3.1 .

Proof. Take time points $t_{1}, \ldots, t_{I}$, let $\mathbf{V}_{N}(t)$ be the vector in (2) in the context of Theorem 3.1, with $G_{j}$ replaced by a finite linear combination of Hermite polynomials (Lemma 4.2). Thus

$$
\mathbf{V}_{N}\left(t_{i}\right)=\left(\sum_{m=k_{1}}^{M} \frac{g_{m, 1}}{A_{1}(N)} S_{N, t_{i}}\left(H_{m}\right), \ldots, \sum_{m=k_{J}}^{M} \frac{g_{m, J}}{A_{J}(N)} S_{N, t_{i}}\left(H_{m}\right)\right) .
$$

We want to show the joint convergence

$$
\left(\mathbf{V}_{N}\left(t_{1}\right), \ldots, \mathbf{V}_{N}\left(t_{I}\right)\right) \stackrel{d}{\rightarrow}\left(\mathbf{B}\left(t_{1}\right), \ldots, \mathbf{B}\left(t_{I}\right)\right)
$$


with $\mathbf{B}(t)$ being the J-dimensional Gaussian process with covariance structure given by (11).

By (6), and because the term $\frac{g_{m, j}}{A_{j}(N)} S_{N, t_{i}}\left(H_{m}\right)$ involves the m-th order Hermite polynomial only, we can represent it as an m-tuple Wiener-Itô integral:

$$
\frac{g_{m, j}}{A_{j}(N)} S_{N, t_{i}}\left(H_{m}\right)=: I_{m}\left(f_{N, m, i, j}\right)
$$

for some square-integrable function $f_{N, m, i, j}$. Now

$$
\mathbf{V}_{N}\left(t_{i}\right)=\left(\sum_{m=k_{1}}^{M} I_{m}\left(f_{N, m, i, 1}\right), \ldots, \sum_{m=k_{J}}^{M} I_{m}\left(f_{N, m, i, J}\right)\right)
$$

To show (27), one only needs to show that as $N \rightarrow \infty,\left(I_{m}\left(f_{N, m, i, j}\right)\right)_{m, i, j}$ converges jointly to a multivariate normal with the correct covariance structure.

Note by the univariate SRD result, namely, Theorem 2.1, each $I_{m}\left(f_{N, m, i, j}\right)=\frac{g_{m, j}}{A_{j}(N)} S_{N, t_{i}}\left(H_{m}\right)$ converges to a univariate normal. Therefore, by Lemma 4.3, it's sufficient to show the covariance structure of $\left(I_{m}\left(f_{N, m, i, j}\right)\right)_{m, i, j}$ is consistent with the covariance structure of $\left(B_{j}\left(t_{i}\right)\right)_{i, j}$ as $N \rightarrow \infty$.

Note that $A_{j}(N)=\sigma_{j} N^{1 / 2}$ where $\sigma_{j}$ is found in (12). If $m_{1} \neq m_{2}$,

$$
E I_{m_{1}}\left(f_{N, m, i_{1}, j_{1}}\right) I_{m_{2}}\left(f_{N, m, i_{2}, j_{2}}\right)=\frac{g_{m_{1}, j_{1}}, g_{m_{2}, j_{2}}}{\sigma_{j_{1}} \sigma_{j_{2}} N} \mathbb{E}\left(S_{N, t_{i_{1}}}\left(H_{m_{1}}\right) S_{N, t_{i_{2}}}\left(H_{m_{2}}\right)\right)=0 .
$$

If $m_{1}=m_{2}=m$,

$$
\begin{aligned}
& \mathbb{E} I_{m}\left(f_{N, m, i_{1}, j_{1}}\right) I_{m}\left(f_{N, m, i_{2}, j_{2}}\right) \\
& =\frac{g_{m, j_{1}}, g_{m, j_{2}}}{\sigma_{j_{1}} \sigma_{j_{2}}} \frac{1}{N} \sum_{n_{1}=1}^{\left[N t_{i_{1}}\right]} \sum_{n_{2}=1}^{\left[N t_{i_{2}}\right]} \mathbb{E}\left(H_{m}\left(X_{n_{1}}\right) H_{m}\left(X_{n_{2}}\right)\right) \\
& =\frac{m ! g_{m, j_{1}}, g_{m, j_{2}}}{\sigma_{j_{1}} \sigma_{j_{2}}} \frac{1}{N} \sum_{n_{1}=1}^{\left[N t_{i_{1}}\right]} \sum_{n_{2}=1}^{\left[N t_{i_{2}}\right]} \gamma\left(n_{1}-n_{2}\right) \\
& \rightarrow \frac{t_{i_{1}} \wedge t_{i_{2}}}{\sigma_{j_{1}} \sigma_{j_{2}}} g_{m, j_{1}}, g_{m, j_{2}} m ! \sum_{n=-\infty}^{\infty} \gamma(n)^{m} \text { as } N \rightarrow \infty
\end{aligned}
$$

by Lemma 4.1 .

Since every component of $\mathbf{V}_{N}$ in (26) is the sum of multiple Wiener-Itô integrals, it follows that

$$
\mathbb{E} V_{N, j_{1}}\left(t_{i_{1}}\right) V_{N, j_{2}}\left(t_{i_{2}}\right) \rightarrow \frac{t_{i_{1}} \wedge t_{i_{2}}}{\sigma_{j_{1}} \sigma_{j_{2}}} \sum_{m=k_{j_{1}} \vee k_{j_{2}}}^{M} g_{m, j_{1}} g_{m, j_{2}} m ! \sum_{n=-\infty}^{\infty} \gamma(n)^{m},
$$

which is the covariance in (11), where here $M$ is finite due to Lemma 4.2 .

\subsection{Proof of Theorem 3.3}

The pure LRD case is proved by extending the proof in [5] to the multivariate case. Set

$$
S_{N, t}(G)=\sum_{n=1}^{[N t]} G\left(X_{n}\right) .
$$

The normalization factor which makes the variance at $t=1$ tend to 1 is

$$
A_{j}(N)=a_{j} L(N)^{k_{j} / 2} N^{1+k_{j}(d-1 / 2)},
$$


where the slowly varying function $L(N)$ stems from the auto-covariance function: $\gamma(n)=L(n) n^{2 d-1}$ and where $a_{j}$ is a normalization constant.

The Hermite expansion of each $G_{j}$ is given in 24 The following reduction lemma shows that it suffices to replace $G_{j}$ 's with corresponding Hermite polynomials.

Lemma 4.4. If the convergence in (13) holds with $g_{k_{j}, j} H_{k_{j}}$ replacing $G_{j}$, then it also holds for $G_{j}, j=$ $1, \ldots, J$.

Proof. By the Cramér-Wold device, we want to show for every $\left(w_{1}, \ldots, w_{J}\right) \in \mathbb{R}^{J}$, the following convergence:

$$
\sum_{j=1}^{J} w_{j} \frac{S_{N, t}\left(G_{j}\right)}{A_{j}(N)} \stackrel{\text { f.d.d. }}{\longrightarrow} \sum_{j=1}^{J} w_{j} Z_{d}^{\left(k_{j}\right)}(t) .
$$

Let $G_{j}^{*}=g_{k_{j}+1, j} H_{k_{j}+1}+g_{k_{j}+2, j} H_{k_{j}+2}+\ldots$, then

$$
\sum_{j=1}^{J} w_{j} \frac{S_{N, t}\left(G_{j}\right)}{A_{j}(N)}=\sum_{j=1}^{J} w_{j} \frac{S_{N, t}\left(g_{k_{j}, j} H_{k_{j}}\right)}{A_{j}(N)}+\sum_{j=1}^{J} w_{j} \frac{S_{N, t}\left(G_{j}^{*}\right)}{A_{j}(N)} .
$$

By the assumption of this lemma and by the Cramér-Wold device,

$$
\sum_{j=1}^{J} w_{j} \frac{S_{N, t}\left(g_{k_{j}, j} H_{k_{j}}\right)}{A_{j}(N)} \stackrel{\text { f.d.d. }}{\longrightarrow} \sum_{j=1}^{J} w_{j} Z_{d}^{\left(k_{j}\right)}(t) .
$$

Hence it suffices to show that for any $t>0$,

$$
\mathbb{E}\left(\sum_{j=1}^{J} w_{j} \frac{S_{N, t}\left(G_{j}^{*}\right)}{A_{j}(N)}\right)^{2} \rightarrow 0 .
$$

By the elementary inequality: $\left(\sum_{j=1}^{J} x_{j}\right)^{2} \leq J \sum_{j=1}^{J} x_{j}^{2}$, it suffices to show that for each $j$,

$$
\mathbb{E}\left(\frac{S_{N, t}\left(G_{j}^{*}\right)}{A_{j}(N)}\right)^{2} \rightarrow 0
$$

This is because the variance growth of $G_{j}^{*}$ (see (7) and (9) $)$ is at most $L_{j}^{*}([N t])[N t]^{\left(k_{j}+1\right)(2 d-1)+2}$ for some slowly varying function $L_{j}^{*}$, while the normalization $A_{j}(N)^{2}=a_{j}^{2} L_{j}(N)^{k_{j}} N^{k_{j}(2 d-1)+2}$ tends more rapidly to infinity.

The following lemma extends Lemma 3 of [ 5 to the multivariate case. It states that if Lemma 3 of [5] holds in the univariate case in each component, then it holds in the multivariate joint case.

Lemma 4.5. Let $F_{0}$ and $F_{N}$ be symmetric locally finite Borel measures without atoms on $\mathbb{R}$ so that $F_{N} \rightarrow F$ weakly. Let $W_{F_{N}}$ and $W_{F_{0}}$ be complex Hermitian Gaussian measures with control measures $F_{N}$ and $F_{0}$ respectively.

Let $K_{N, j}$ be a series of Hermitian $(K(-\mathbf{x})=\overline{K(\mathbf{x})})$ measurable functions of $k_{j}$ variables tending to a continuous function $K_{0, j}$ uniformly in any compact set in $\mathbb{R}^{k_{j}}$ as $N \rightarrow \infty$.

Moreover, suppose the following uniform integrability type condition holds for every $j=1, \ldots, J$ :

$$
\lim _{A \rightarrow \infty} \sup _{N} \int_{\mathbb{R}^{k_{j}} \backslash[-A, A]^{k_{j}}}\left|K_{N, j}(\mathbf{x})\right|^{2} F_{N}\left(d x_{1}\right), \ldots, F_{N}\left(d x_{k_{j}}\right)=0 .
$$

Then we have the joint convergence:

$$
\left(I_{k_{1}}^{(N)}\left(K_{N, 1}\right), \ldots, I_{k_{J}}^{(N)}\left(K_{N, J}\right)\right) \stackrel{d}{\rightarrow}\left(I_{k_{1}}^{(0)}\left(K_{0,1}\right), \ldots, I_{k_{J}}^{(0)}\left(K_{0, J}\right)\right) .
$$

where $I_{k}^{(N)}($.$) denotes a k$-tuple Wiener-Itô integral with respect to complex Gaussian random measure $W_{F_{N}}$, $N=0,1,2, \ldots$ 
Proof. By the Cramér-Wold device, we need to show that for every $\left(w_{1}, \ldots, w_{J}\right) \in \mathbb{R}^{J}$ as $N \rightarrow \infty$,

$$
X_{N}:=\sum_{j=1}^{J} w_{j} I_{k_{j}}^{(N)}\left(K_{N, j}\right) \stackrel{d}{\rightarrow} X_{0,0}:=\sum_{j=1}^{J} w_{j} I_{k_{j}}^{(0)}\left(K_{0, j}\right) .
$$

We show first that (32) holds when replacing all kernels with simple Hermitian functions $g_{j}$ of the form:

$$
g_{j}\left(u_{1}, \ldots, u_{k_{j}}\right)=\sum_{i_{1}, \ldots, i_{k}=1}^{n} a_{i_{1}, \ldots, i_{k_{j}}} 1_{A_{i_{1}, j} \times \ldots \times A_{i_{k_{j}}, j}}\left(u_{1}, \ldots, u_{k_{j}}\right),
$$

where $A_{i, j}$ 's are bounded Borel sets in $\mathbb{R}$ satisfying $F_{0}\left(\partial A_{i, j}\right)=0, a_{i_{1}, \ldots, i_{k_{j}}}=0$ if any two of $i_{1}, \ldots, i_{k_{j}}$ are equal, and $\overline{g(\mathbf{u})}=g(-\mathbf{u})$. We claim that

$$
\sum_{j=1}^{s} w_{j} I_{k_{j}}^{(N)}\left(g_{j}\right) \stackrel{d}{\rightarrow} \sum_{j=1}^{s} w_{j} I_{k_{j}}^{(0)}\left(g_{j}\right)
$$

Indeed, since $F_{N} \rightarrow F_{0}$ weakly and $F_{0}\left(\partial A_{i, j}\right)=0$, we have as $N \rightarrow \infty$ :

$$
\mathbb{E} W_{F_{N}}\left(A_{i, j}\right) W_{F_{N}}\left(A_{k, l}\right)=F_{N}\left(A_{i, j} \cap A_{k, l}\right) \rightarrow F_{0}\left(A_{i, j} \cap A_{k, l}\right)=\mathbb{E} W_{F_{0}}\left(A_{i, j}\right) W_{F_{N}}\left(A_{k, l}\right),
$$

thus $\left(W_{F_{N}}\left(A_{i, j}\right)\right)_{i, j} \stackrel{d}{\rightarrow}\left(W_{F_{0}}\left(A_{i, j}\right)\right)_{i, j}$ jointly. Since $\sum_{j=1}^{s} w_{j} I_{k_{j}}^{(N)}\left(g_{j}\right)$ is a polynomial of $W_{F_{N}}\left(A_{i, j}\right)$ and by Continuous Mapping Theorem, (33) holds.

Next, due to the atomlessness of $F_{N}$, the uniform convergence of $K_{N, j}$ to $K_{0, j}$ on any compact set, (30) and the continuity of $K_{0, j}$, for any $\epsilon>0$, there exist simple Hermitian $g_{j}$ 's $j=1, \ldots, J$ as above, such that for $N=0$ and $N>N(\epsilon)$ (large enough),

$$
\int_{\mathbb{R}^{k_{j}}}\left|K_{N, j}\left(x_{1}, \ldots, x_{k_{j}}\right)-g_{j}\left(x_{1}, \ldots, x_{k_{j}}\right)\right|^{2} F_{N}\left(d x_{1}\right) \ldots F_{N}\left(d x_{k_{j}}\right)<\epsilon .
$$

By (34) for every $j=1, \ldots, J$, we can find a sequence $g_{M, j}$ such that

$$
\begin{gathered}
\left\|I_{k_{j}}^{(0)}\left(K_{0, j}\right)-I_{k_{j}}^{(0)}\left(g_{M, j}\right)\right\|_{L^{2}}<1 / M \\
\left\|I_{k_{j}}^{N}\left(K_{N, j}\right)-I_{k_{j}}^{N}\left(g_{j}\right)\right\|_{L^{2}}<1 / M \text { for } N>N(M) \text { (large enough), }
\end{gathered}
$$

hence by (35)

$$
X_{0, M}:=\sum_{j=1}^{J} w_{j} I_{k_{j}}^{(0)}\left(g_{M, j}\right) \stackrel{d}{\rightarrow} X_{0,0}:=\sum_{j=1}^{J} w_{j} I_{k_{j}}^{(0)}\left(K_{0}\right) \quad \text { as } M \rightarrow \infty .
$$

and by (36),

$$
\begin{aligned}
& \lim _{M} \limsup _{N} \mathbb{E}\left|X_{N}-X_{N, M}\right|^{2} \\
:= & \lim _{M} \limsup _{N} \mathbb{E}\left|\sum_{j=1}^{J} w_{j} I_{k_{j}}^{(N)}\left(K_{N, j}\right)-\sum_{j=1}^{J} w_{j} I_{k_{j}}^{(N)}\left(g_{M, j}\right)\right|^{2}=0 .
\end{aligned}
$$

Finally, replacing $g_{j}$ by $g_{M, j}$ in (33), we have

$$
X_{N, M} \stackrel{d}{\rightarrow} X_{0, M}
$$

Thus (32), namely, $X_{N} \stackrel{d}{\rightarrow} X_{0,0}$, follows now from (37), (38) and (39) and Theorem 3.2 of [2].

We can now prove Theorem 3.3:

Proof. Since Lemma 4.5 involves only univariate assumptions and concludes with the desired multivariate convergence (31), one needs to treat only the univariate case. This is done in [5]. 


\subsection{Proof of Theorem 3.6}

The following result from [16] will be used:

Theorem 4.6. (Theorem 4.7 in [16].) Consider

$$
\begin{aligned}
& \mathbf{S}_{N}=\left(I_{k_{1, S}}\left(f_{1, S, N}\right), \ldots, I_{k_{J_{S}, S}}\left(f_{J_{S}, S, N}\right)\right), \\
& \mathbf{L}_{N}=\left(I_{k_{1, L}}\left(f_{1, L, N}\right), \ldots, I_{k_{J_{L}, L}}\left(f_{J_{L}, L, N}\right)\right),
\end{aligned}
$$

where $k_{j_{S}, S}>k_{j_{L}, L}$ for all $j_{S}=1, \ldots, J_{S}$ and $j_{L}=1, \ldots, J_{L}$.

Suppose that as $N \rightarrow \infty, \mathbf{S}_{N}$ converges in distribution to a multivariate normal law, and $\mathbf{L}_{N}$ converges in distribution to a multivariate law which has moment-determinate components, then there are independent random vectors $\mathbf{Z}$ and $\mathbf{H}$, such that

$$
\left(\mathbf{S}_{N}, \mathbf{L}_{N}\right) \stackrel{d}{\rightarrow}(\mathbf{Z}, \mathbf{H}) .
$$

A proof of Theorem 4.6 can be found in Appendix B (see Theorem B.3).

Proof of Theorem 3.6. Using the reduction arguments of Lemma 4.2 and Lemma 4.4, we can replace $G_{j, S}$ in (16) with $\sum_{m=k_{j, S}}^{M} g_{m, j, S} H_{m}$, and we can replace $G_{j, L}$ in (17) with $g_{k_{L}, j, L} H_{k_{L}}$, where $k_{j, S}>k_{j, L}=1$ or 2 are the corresponding Hermite ranks and $g_{m, j, S}, g_{k_{L}, j, L}$ are the corresponding coefficients of their Hermite expansions.

Fix finite time points $t_{i}, i=1 \ldots, I$, we need to consider the joint convergence of the following vector:

$$
\left(S_{i, j_{S}, N}, L_{i, j_{L}, N}\right)_{i, j_{S}, j_{L}}:=\left(\frac{1}{A_{j_{S}, S}} \sum_{m=k_{j_{S}, S}}^{M} g_{m, j_{S}, S} S_{N, t_{i}}\left(H_{m}\right), \frac{1}{A_{j_{L}, L}} g_{k_{L}, j_{L}, L} S_{N, t_{i}}\left(H_{k_{L}}\right)\right)_{i, j_{S}, j_{L}},
$$

where $i=1, \ldots, I, j_{S}=1, \ldots, J_{S}, j_{L}=1, \ldots, J_{L}$.

As in the proof of Theorem 3.1, using (6), we express Hermite polynomials as multiple Wiener-Itô integrals:

$$
S_{i, j_{S}, N}=\sum_{m=k_{j_{S}, S}}^{M} I_{m}\left(f_{m, i, j_{S}, N}\right), \quad L_{i, j_{L}, N}=\sum_{m=k_{j_{L}, L}}^{M} I_{m}\left(f_{m, i, j_{L}, N}\right),
$$

where $f_{m, i, j_{S}, N}, f_{i, j_{L}, N}$ are some symmetric square-integrable functions.

Express the vector in (40) as $\left(\mathbf{S}_{N}, \mathbf{L}_{N}\right)$, where $\mathbf{S}_{N}:=\left(S_{i, j_{S}, N}\right)_{i, j_{S}}, \mathbf{L}_{N}:=\left(L_{i, j_{L}, N}\right)_{i, j_{L}}$.

By Theorem 3.1, $\mathbf{S}_{N}$ converges in distribution to some multivariate normal distribution, and by Theorem $3.3, \mathbf{L}_{N}$ converges to a multivariate distribution with moment-determinate marginals, because by assumption the limits only involve Hermite rank $k=1$ (normal distribution) and $k=2$ (Rosenblatt distribution). The normal distribution is moment-determinate. The Rosenblatt distribution is also momentdeterminate because it has analytic characteristic function (23] p.301).

We can now use Theorem 4.6 to conclude the proof.

\section{A Invariance of joint distribution among different representations of Hermite process}

The Hermite process admits four different representations ([21]):

Let $B($.$) be the real Gaussian random measure and W($.$) be the complex Gaussian random measure, as$ defined in Section 6 of [24]. $H_{0} \in(1-1 /(2 k), 1)$. 
1. Time domain representation:

$$
Z_{H_{0}}^{(k)}(t)=a_{k, H_{0}}=\int_{\mathbb{R}^{k}}^{\prime}\left(\int_{0}^{t} \prod_{j=1}^{k}\left(s-x_{j}\right)_{+}^{H_{0}-3 / 2} d s\right) B\left(d x_{1}\right) \ldots B\left(d x_{k}\right)
$$

2. Spectral domain representation:

$$
Z_{H_{0}}^{(k)}(t)=b_{k, H_{0}} \int_{\mathbb{R}^{k}}^{\prime \prime} \frac{e^{i\left(x_{1}+\ldots+x_{k}\right) t}-1}{i\left(x_{1}+\ldots+x_{k}\right)} \prod_{j=1}^{k}\left|x_{j}\right|^{1 / 2-H_{0}} W\left(d x_{1}\right) \ldots W\left(d x_{k}\right)
$$

3. Positive half-axis representation:

$$
Z_{H_{0}}^{(k)}(t)=c_{k, H_{0}} \int_{[0, \infty)^{k}}^{\prime}\left(\int_{0}^{t} \prod_{j=1}^{k} x_{j}^{1 / 2-H_{0}}\left(1-s x_{j}\right)_{+}^{H_{0}-3 / 2} d s\right) B\left(d x_{1}\right) \ldots B\left(d x_{k}\right)
$$

4. Finite interval representation:

$$
Z_{H_{0}}^{(k)}(t)=d_{k, H_{0}} \int_{[0, t]^{k}}^{\prime}\left(\prod_{j=1}^{k} x_{j}^{1 / 2-H_{0}} \int_{0}^{t} x^{k\left(H_{0}-1 / 2\right)} \prod_{j=1}^{k}\left(s-x_{j}\right)_{+}^{H_{0}-3 / 2} d s\right) B\left(d x_{1}\right) \ldots B\left(d x_{k}\right)
$$

where $a_{k, H_{0}}, b_{k, H_{0}}, c_{k, H_{0}}, d_{k, H_{0}}$ are constant coefficients to guarantee that $\operatorname{Var}\left(Z_{H_{0}}^{(k)}(t)\right)=1$, given in $(1.17)$ and (1.18) of [21].

Keep $H_{0}$ fixed throughout. We will prove the following:

Theorem A.1. The joint distribution of a vector made up of Hermite processes of possibly different orders $k$, but sharing the same random measure $B($.$) or W($.$) in their Wiener-Itô integral representations, remains$ the same when switching from one of the above representations to another.

The following notations are used to denote Wiener-Itô integrals with respect to $B($.$) and W($.$) respectively:$

$$
\begin{gathered}
I(f):=\int_{\mathbb{R}^{k}}^{\prime} f\left(x_{1}, \ldots, x_{k}\right) d B\left(x_{1}\right) \ldots d B\left(x_{k}\right), \\
\tilde{I}(g):=\int_{\mathbb{R}^{k}}^{\prime \prime} g\left(\omega_{1}, \ldots, \omega_{k}\right) d W\left(\omega_{1}\right) \ldots d W\left(\omega_{k}\right) .
\end{gathered}
$$

where ' indicates that we don't integrate on $x_{i}=x_{j}, i \neq j$, " indicates that we don't integrate on $\omega_{i}= \pm \omega_{j}$, $i \neq j, f$ is a symmetric function and $g$ is an Hermitian function $(g(\omega)=\overline{g(-\omega)})$.

The next lemma establishes the equality in joint distribution between time domain representation (41) and spectral domain representation (42), which is a multivariate extension of Lemma 6.1 in [24].

Lemma A.2. Suppose that $A_{j}\left(x_{1}, \ldots, x_{k_{j}}\right)$ is a symmetric function in $L^{2}\left(\mathbb{R}^{k_{j}}\right), j=1, \ldots, J$. Let $\tilde{A}\left(x_{1}, \ldots, x_{k_{j}}\right)$ be its $L^{2}$-Fourier transform:

$$
\tilde{A}_{j}\left(\omega_{1}, \ldots, \omega_{k_{j}}\right)=\frac{1}{(2 \pi)^{k_{j} / 2}} \int_{\mathbb{R}^{m}} \exp \left(i \sum_{n=1}^{k_{j}} x_{n} \omega_{n}\right) A_{j}\left(x_{1}, \ldots, x_{k_{j}}\right) d x_{1} \ldots d x_{k_{j}} .
$$

Then

$$
\left(I_{k_{1}}\left(A_{1}\right), \ldots, I_{k_{J}}\left(A_{J}\right)\right) \stackrel{d}{=}\left(\tilde{I}_{k_{1}}\left(\tilde{A}_{1}\right), \ldots, \tilde{I}_{k_{J}}\left(\tilde{A}_{J}\right)\right)
$$


Proof. The proof is a slight extension of the proof of Lemma 6.1 of 24. The idea is to use a complete orthonormal set $\left\{\psi_{i}, i \geq 0\right\}$ in $L^{2}(\mathbb{R})$ to represent each $A_{j}$ as an infinite polynomial form of order $k_{j}$ with respect to $\psi_{i}$ 's, as is done in (6.3) of [24]. Each $I_{k_{j}}\left(A_{j}\right)$ can be then written in the form of (6.4) of [24], which is essentially a function of $X_{i}:=\int \psi_{i}(x) d B(x), i \geq 0$, denoted

$$
I_{k_{j}}\left(A_{j}\right)=K_{j}(\mathbf{X})
$$

where $\mathbf{X}=\left(X_{0}, X_{1}, \ldots\right)$. Thus

$$
\left(I_{k_{1}}\left(A_{1}\right), \ldots, I_{k_{J}}\left(A_{J}\right)\right)=\mathbf{K}(\mathbf{X}),
$$

where the vector function $\mathbf{K}=\left(K_{1}, \ldots, K_{J}\right)$.

Now, $\tilde{A}_{j}$ can also be written as an infinite polynomial form of order $k_{j}$ with respect to $\tilde{\psi}_{i}, i \geq 0$, where $\tilde{\psi}_{i}(\omega)=(2 \pi)^{-1 / 2} \int e^{i x \omega} \psi_{i}(x) d x$ is the $L^{2}$-Fourier transform of $\psi_{i}$, as is done in (6.5) of [24]. Set $Y_{j}:=\int \tilde{\psi}_{i}(\omega) d W(\omega), i \geq 0$. Then, as in (6.6) of [24, we have

$$
\tilde{I}_{k_{j}}\left(\tilde{A}_{j}\right)=K_{j}(\mathbf{Y}),
$$

where $K_{j}$ 's are the same as above, $\mathbf{Y}=\left(Y_{0}, Y_{1}, \ldots\right)$, and thus

$$
\left(\tilde{I}_{k_{1}}\left(\tilde{A}_{1}\right), \ldots, \tilde{I}_{k_{J}}\left(\tilde{A}_{J}\right)\right)=\mathbf{K}(\mathbf{Y})
$$

By (45) and (46), it suffices to show that $\mathbf{X} \stackrel{d}{=} \mathbf{Y}$. This is true because by Parseval's identity, $\mathbf{X}$ and $\mathbf{Y}$ both consist of i.i.d. normal random variables with mean 0 and identical variance, . For details, see [24].

We now complete the proof of Theorem A.1. We still need to justify the equality in joint distribution between time domain representation (41) and positive half-axis representation (43) or finite interval representation (44).

First let's summarize the arguments of 21] for going from (41) to (43) or (44). The heuristic idea is that by changing the integration order in (41), one would have

$$
\begin{aligned}
Z_{H_{0}}^{(k)} & =\int_{0}^{t}\left(\int_{\mathbb{R}^{k}}^{t} \prod_{j=1}^{k}\left(s-x_{j}\right)^{H_{0}-3 / 2} B\left(d x_{1}\right) \ldots B\left(d x_{k}\right)\right) d s \\
& =\int_{0}^{t} H_{k}\left(\int_{\mathbb{R}}(s-x)_{+}^{H_{0}-3 / 2} B(d x)\right) d s,
\end{aligned}
$$

where $H_{k}$ is $k$-th Hermite polynomial. But in fact $g(x):=(s-x)_{+}^{H_{0}-3 / 2} \notin L^{2}(\mathbb{R})$, and consequently $G(s):=\int_{\mathbb{R}}(s-x)_{+}^{H_{0}-3 / 2} B(d x)$ is not well-defined.

The way to get around this is to do a regularization, that is, to truncate $g(x)$ as $g_{\epsilon}(x):=g(x) 1_{s-x>\epsilon}(x)$ for $\epsilon>0$. Now the Gaussian process $G_{\epsilon}(t):=\int_{\mathbb{R}} g_{\epsilon}(x) B(d x)$ is well-defined. Next, after some change of variables, one gets the new desired representation of $G_{\epsilon}(t)$, say $G_{\epsilon}^{*}(t)$, where $G_{\epsilon}^{*}(t) \stackrel{d}{=} G_{\epsilon}(t)$. Setting $Z_{\epsilon, H_{0}}^{(k)}(t)=\int_{0}^{t} H_{k}\left(G_{\epsilon}(t)\right) d t$ and $Z_{\epsilon, H_{0}}^{(k) *}(t)=\int_{0}^{t} H_{k}\left(G_{\epsilon}^{*}(t)\right) d t$, yields

$$
Z_{\epsilon, H_{0}}^{(k)}(t) \stackrel{d}{=} Z_{\epsilon, H_{0}}^{(k) *}(t) .
$$

Finally by letting $\epsilon \rightarrow 0$, one can show that $Z_{\epsilon, H_{0}}^{(k)}(t)$ converges in $L^{2}(\Omega)$ to the Hermite process $Z_{H_{0}}^{(k)}(t)$, while $Z_{\epsilon, H_{0}}^{(k) *}(t)$ converges in $L^{2}(\Omega)$ to some $Z_{H_{0}}^{(k) *}(t)$, which is the desired alternative representation of $Z_{H_{0}}^{(k)}(t)$.

The above argument relies on the stochastic Fubini theorem (Theorem 2.1 of [21]) which legitimates the change of integration order, that is, for $f(s, \mathbf{x})$ defined on $\mathbb{R} \times \mathbb{R}^{k}$, if $\int_{\mathbb{R}}\|f(s, .)\|_{L^{2}\left(\mathbb{R}^{k}\right)} d s<\infty$ (which is the case after regularization), then

$$
\int_{\mathbb{R}^{k}}^{\prime} \int_{\mathbb{R}} f\left(s, x_{1}, \ldots, x_{k}\right) d s B\left(d x_{1}\right) \ldots B\left(d x_{k}\right)=\int_{\mathbb{R}} \int_{\mathbb{R}^{k}}^{\prime} f\left(s, x_{1}, \ldots, x_{k}\right) B\left(d x_{1}\right) \ldots B\left(d x_{k}\right) d s \quad \text { a.s. }
$$


Now, consider the multivariate case. Note that we still have equality of the the joint distributions as in (48) and the equality is preserved in the $L^{2}(\Omega)$ limit as $\epsilon \rightarrow 0$. Moreover, the stochastic Fubini theorem (Theorem 2.1 of 21]) extends naturally to the multivariate setting since the change of integration holds as an almost sure equality. Therefore one gets equality in joint distribution when switching from (41) to (43) or (44).

\section{B Asymptotic independence of Wiener-Itô integral vectors}

We prove here Theorem 4.1 by extending a combinatorial proof of Nourdin and Rosinski [15] 11 .

First, some background. In the papers [25] and [10], a criterion for independence between two random variables belonging to Wiener Chaos, say, $I_{p}(f)$ and $I_{p}(g)$, is given as

$$
f \otimes_{1} g=0 \quad \text { a.s. }
$$

where $\otimes_{1}$ means contraction of order 1 and is defined below.

The result of [16] involves the following problem: if one has sequences $\left\{f_{n}\right\},\left\{g_{n}\right\}$, when will asymptotic independence hold between $I_{p}\left(f_{n}\right)$ and $I_{q}\left(g_{n}\right)$ as $n \rightarrow \infty$ ? Motivated by (49), one may guess that the criterion is $f_{n} \otimes_{1} g_{n} \rightarrow 0$ as $n \rightarrow \infty$. This is, however, shown to be false by a counterexample in [16: set $p=q=2$, $f_{n}=g_{n}$ and assume that $I_{2}\left(f_{n}\right) \stackrel{d}{\rightarrow} Z \sim N(0,1)$. One can then show that $f_{n} \otimes_{1} f_{n} \rightarrow 0$, while obviously $\left(I_{2}\left(f_{n}\right), I_{2}\left(f_{n}\right)\right) \stackrel{d}{\rightarrow}(Z, Z)$. Let $\|$.$\| denote the L^{2}$ norm in the appropriate dimension and let $<., .>$ denote the corresponding inner product.

We now define contractions. The contraction $\otimes_{r}$ between two symmetric square integrable functions $f$ and $g$ is defined as

$$
\begin{aligned}
& \left(f \otimes_{r} g\right)\left(x_{1}, \ldots, x_{p-r}, y_{1}, \ldots, y_{q-r}\right):= \\
& \int_{\mathbb{R}^{r}} f\left(x_{1}, \ldots, x_{p-r}, s_{1}, \ldots, s_{r}\right) g\left(y_{1}, \ldots, y_{q-s}, s_{1}, \ldots, s_{r}\right) d s_{1} \ldots d s_{r}
\end{aligned}
$$

If $r=0$, the contraction is just the tensor product:

$$
f \otimes_{0} g=f \otimes g:=f\left(x_{1}, \ldots, x_{p}\right) g\left(y_{1}, \ldots, y_{q}\right) .
$$

The symmetrized contraction $\tilde{\otimes}_{r}$ involves one more step, namely, the symmetrization of the function obtained from the contraction. This is done by summing over all permutations of the variables and dividing by the number of permutations. Note that as the contraction is only defined for symmetric functions, replacing $\otimes_{r}$ with $\tilde{\otimes}_{r}$ enables one to consider a sequence of symmetrized contractions of the form $\left(\ldots\left(\left(f_{1} \tilde{\otimes}_{r_{1}} f_{2}\right) \tilde{\otimes}_{r_{2}} f_{3}\right) \ldots\right) \tilde{\otimes}_{r_{n-1}} f_{n}$.

We will use the following product formula (Proposition 6.4.1 of [18]) for multiple Wiener-Itô integrals

$$
I_{p}(f) I_{q}(g)=\sum_{r=0}^{p \wedge q} r !\left(\begin{array}{l}
p \\
r
\end{array}\right)\left(\begin{array}{l}
q \\
r
\end{array}\right) I_{p+q-2 r}\left(f \otimes_{r} g\right) \quad p, q \geq 0 .
$$

Because the symmetrization of the integrand doesn't change the multiple Wiener-Itô integral, $\otimes_{r}$ could be replaced with $\tilde{\otimes}_{r}$ in the product formula.

For a vector $\mathbf{q}=\left(q_{1}, \ldots, q_{k}\right)$, we denote $|\mathbf{q}|:=q_{1}+\ldots+q_{k}$. By a suitable iteration of (51), we have the following multiple product formula:

$$
\prod_{i=1}^{k} I_{q_{i}}\left(f_{i}\right)=\sum_{\mathbf{r} \in C(\mathbf{q}, k)} a(\mathbf{q}, k, \mathbf{r}) I_{|\mathbf{q}|-2|\mathbf{r}|}\left(\ldots\left(f_{1} \tilde{\otimes}_{r_{1}} f_{2}\right) \ldots \tilde{\otimes}_{r_{k-1}} f_{k}\right),
$$

\footnotetext{
${ }^{1}$ The proof in Theorem B.1 below is an extension to Wiener-Itô integral vectors of the original combinatorial proof of Theorem 3.1 of [15] given for Wiener-Itô integral scalars. The result also follows from Theorem 3.4 in [16] which includes Wiener-Itô integral vectors, but with a proof based on Malliavin Calculus.
} 
where $\mathbf{q} \in \mathbb{N}^{n}$, the index set $C(\mathbf{q}, k)=\left\{\mathbf{r} \in \prod_{i=1}^{k-1}\left\{0,1, \ldots, q_{i+1}\right\}: r_{1} \leq q_{1}, r_{i} \leq\left(q_{1}+\ldots+q_{i}\right)-2\left(r_{1}+\ldots+\right.\right.$ $\left.\left.r_{i-1}\right), i=2, \ldots k-1\right\}$, and $a(\mathbf{q}, k, \mathbf{r})$ is some integer factor.

Theorem B.1. (Asymptotic Independence of Multiple Wiener-Itô Integral Vectors.) Suppose we have the joint convergence

$$
\left(\mathbf{U}_{1, N}, \ldots, \mathbf{U}_{J, N}\right) \stackrel{d}{\rightarrow}\left(\mathbf{U}_{1}, \ldots, \mathbf{U}_{J}\right),
$$

where

$$
\mathbf{U}_{j, N}=\left(I_{q_{1, j}}\left(f_{1, j, N}\right), \ldots, I_{q_{I_{j}, j}}\left(f_{I_{j}, j, N}\right)\right)
$$

Assume

$$
\lim _{N \rightarrow \infty}\left\|f_{i_{1}, j_{1}, N} \otimes_{r} f_{i_{1}, j_{2}, N}\right\|=0
$$

for all $i_{1}, i_{2}, j_{1} \neq j_{2}$, and $r=1, \ldots, q_{i_{1}, j_{1}} \wedge q_{i_{2}, j_{2}}$.

Then using the notation $\mathbf{u}^{\mathbf{k}}=u_{1}^{k_{1}} \ldots u_{m}^{k_{m}}$, we have

$$
\mathbb{E}\left[\mathbf{U}_{1}^{\mathbf{k}_{1}} \ldots \mathbf{U}_{J}^{\mathbf{k}_{J}}\right]=\mathbb{E}\left[\mathbf{U}_{1}^{\mathbf{k}_{1}}\right] \ldots \mathbb{E}\left[\mathbf{U}_{J}^{\mathbf{k}_{J}}\right]
$$

for all $\mathbf{k}_{j} \in \mathbb{N}^{I_{j}}$

Moreover, if every component of every $\mathbf{U}_{j}$ is moment-determinate, then $\mathbf{U}_{1}, \ldots, \mathbf{U}_{J}$ are independent.

Proof. This is an extension of a proof in 15.

The index $i=1, \ldots, I_{j}$ refers to the components within the vector $\mathbf{U}_{j, N}, j=1, \ldots, J$. For notational simplicity, we let $I_{j}=I$, that is, each $\mathbf{U}_{j, N}$ has the same number of components.

Let $|\mathbf{k}|$ denote the sum of its components $k_{1}+\ldots+k_{m}$. First to show (54), it suffices to show

$$
\lim _{N \rightarrow \infty} \mathbb{E} \prod_{j=1}^{J}\left(\mathbf{U}_{j, N}^{\mathbf{k}_{j}}-\mathbb{E}\left[\mathbf{U}_{j, N}^{\mathbf{k}_{j}}\right]\right)=0
$$

for any $\left|\mathbf{k}_{1}\right|>0, \ldots,\left|\mathbf{k}_{\mathbf{J}}\right|>0$. Note that $\mathbf{U}_{j, N}^{\mathbf{k}_{j}}=U_{1, j, N}^{k_{1, j}} \ldots U_{I, j, k}^{k_{I, j}}$ is a scalar.

By (152), one gets

$$
I_{q}(f)^{k}=\sum_{\mathbf{r} \in C_{q, k}} a(q, k, \mathbf{r}) I_{k q-2|\mathbf{r}|}\left(\ldots\left(f \tilde{\otimes}_{r_{1}} f\right) \ldots \tilde{\otimes}_{r_{k-1}} f\right)
$$

where $a(q, k, r)$ 's are integer factors which don't play an important role, and $C_{q, k}$ is some index set. If $\mathbf{U}_{j, N}^{\mathbf{k}_{j}}=\prod_{i=1}^{I} I_{q_{i, j}}\left(f_{i, j, N}\right)^{k_{i, j}}$, then

$$
\begin{aligned}
\mathbf{U}_{j, N}^{\mathbf{k}_{j}} & =\prod_{i=1}^{I} \sum_{\mathbf{r} \in C_{q_{i, j}, k_{i, j}}} a\left(q_{i, j}, k_{i, j}, \mathbf{r}\right) I_{k_{i, j} q_{i, j}-2|\mathbf{r}|}\left(\ldots\left(f_{i, j, N} \tilde{\otimes}_{r_{1}} f_{i, j, N}\right) \ldots \tilde{\otimes}_{r_{k_{i, j}-1}} f_{i, j, N}\right) \\
& =\sum_{\mathbf{r}^{1} \in C_{q_{1, j}, k_{1, j}}} \ldots \sum_{\mathbf{r}^{I} \in C_{q_{I, j}, k_{I, j}}} \prod_{i=1}^{I} a\left(q_{i, j}, k_{i, j}, \mathbf{r}^{i}\right) I_{k_{i, j} q_{i, j}-2\left|\mathbf{r}^{i}\right|}\left(h_{i, j, N}\right)
\end{aligned}
$$

where

$$
h_{i, j, N}=\left(\ldots\left(f_{i, j, N} \tilde{\otimes}_{r_{1}^{i}} f_{i, j, N}\right) \ldots \tilde{\otimes}_{r_{i, j}^{i}-1} f_{i, j, N}\right) .
$$

If one applies the product formula (52) to the product in (55), one gets that $\mathbf{U}_{j, N}^{\mathbf{k}_{j}}$ involves terms of the form $I_{\left|\mathbf{p}_{j}\right|-2\left|\mathbf{s}_{j}\right|}\left(H_{j, N}\right)\left(\mathbf{p}_{j}\right.$ and $\mathbf{s}_{j}$ run through some suitable index sets), where

$$
H_{j, N}=\left(\ldots\left(h_{1, j, N} \tilde{\otimes}_{s_{1}} h_{2, j, N}\right) \ldots \tilde{\otimes}_{s_{I-1}} h_{I, j, N}\right) .
$$

Since the expectation of a Wiener-Itô integral of positive order is 0 while a Wiener-Itô integral of zero order is a constant, $\mathbf{U}_{j, N}^{\mathbf{k}_{j}}-\mathbb{E}\left[\mathbf{U}_{j, N}^{\mathbf{k}_{j}}\right]$ involves $I_{\left|\mathbf{p}_{j}\right|-2\left|\mathbf{s}_{j}\right|}\left(H_{j, N}\right)$ with $\left|\mathbf{p}_{j}\right|-2\left|\mathbf{s}_{j}\right|>0$ only. Therefore, every $H_{j, N}$ involved in the expression of $\mathbf{U}_{j, N}^{\mathbf{k}_{j}}-\mathbb{E}\left[\mathbf{U}_{j, N}^{\mathbf{k}_{j}}\right]$ has $n_{j}=\left|\mathbf{p}_{j}\right|-2\left|\mathbf{s}_{j}\right|>0$ variables. 
Note that there are no products left at this point in the expression of $\mathbf{U}_{j, N}^{\mathbf{k}_{j}}-\mathbb{E}\left[\mathbf{U}_{j, N}^{\mathbf{k}_{j}}\right]$, only sums. But to compute $\mathbb{E} \prod_{j=1}^{J}\left(\mathbf{U}_{j, N}^{\mathbf{k}_{j}}-\mathbb{E}\left[\mathbf{U}_{j, N}^{\mathbf{k}_{j}}\right]\right)$, one needs to apply the product formula (52) again and then compute the expectation. Since Wiener-Itô integrals of positive order have mean 0 , taking the expectation involves focusing on the terms of zero order which are constants. Since $f \otimes_{p} g=<f, g>=E I_{p}(f) I_{p}(g)$ for functions $f$ and $g$ both having $p$ variables, $\mathbb{E} \prod_{j=1}^{J}\left(\mathbf{U}_{j, N}^{\mathbf{k}_{j}}-\mathbb{E}\left[\mathbf{U}_{j, N}^{\mathbf{k}_{j}}\right]\right)$ involves only terms of the form:

$$
\begin{aligned}
G_{N} & =\left(\ldots\left(H_{1, N} \tilde{\otimes}_{t_{1}} H_{2, N}\right) \ldots \tilde{\otimes}_{t_{J-2}} H_{J-1, N}\right) \tilde{\otimes}_{t_{J-1}} H_{J, N} \\
& \left.=\int_{\mathbb{R}^{n} J}\left(H_{1, N} \tilde{\otimes}_{t_{1}} H_{2, N}\right) \ldots \tilde{\otimes}_{t_{J-2}} H_{J-1, N}\right) H_{J, N} d \mathbf{x}
\end{aligned}
$$

where the contraction size vector $\mathbf{t}=\left(t_{1}, \ldots, t_{J-1}\right)$ runs through some index set. Since these contractions must yield a constant, we have

$$
|\mathbf{t}|=\frac{1}{2}\left(n_{1}+\ldots+n_{J}\right)>0
$$

where $n_{j}$ is the number of variables of $H_{j, N}$. There is therefore at least one component (call it $t$ ) of $\mathbf{t}$ which is strictly positive and thus there is a pair $j_{1}, j_{2}$ with $j_{1} \neq j_{2}$, such that $H_{J_{1}}$ and $H_{j_{2}}$ that have at least one common argument.

One now needs to show that $G_{N}$ in (57) tends to 0 . This is done by applying the generalized CauchySchwartz inequalities in Lemma 2.3 of [16] successively, through the following steps:

$$
\begin{aligned}
& \text { for any } j_{1} \neq j_{2}, i_{1}, i_{2} \text { and } r>0, \lim _{N \rightarrow \infty}\left\|f_{i_{1}, j_{1}, N} \otimes_{r} f_{i_{2}, j_{2}, N}\right\|=0 \\
& \Longrightarrow \text { for any } j_{1} \neq j_{2}, i_{1}, i_{2} \text { and } s>0, \lim _{N \rightarrow \infty}\left\|h_{i_{1}, j_{1}, N} \otimes_{s} h_{i_{2}, j_{2}, N}\right\|=0 \\
& \Longrightarrow \text { for any } j_{1} \neq j_{2} \text { and } t>0, \lim _{N \rightarrow \infty}\left\|H_{j_{1}, N} \otimes_{t} H_{j_{2}, N}\right\|=0 \\
& \Longrightarrow \lim _{N \rightarrow \infty} G_{N}=0,
\end{aligned}
$$

proving (54). Here we illustrate some details for going from (59) to (60), and omit the first two steps which use a similar argument.

Let $C=\left\{1,2, \ldots,\left(n_{1}+\ldots n_{J}\right) / 2\right\}$. Suppose $c$ is a subset of $C$, then we use the notation $\mathbf{z}_{c}$ to denote $\left\{z_{j_{1}}, \ldots, z_{j_{|c|}}\right\}$ where $\left\{j_{1}, \ldots, j_{|c|}\right\}=c$ and $|c|$ is the cardinality of $c$. When $c=\emptyset, \mathbf{z}_{c}=\emptyset$.

Observe that (57) is a sum (due to symmetrization) of terms of the form:

$$
\int_{\mathbb{R}^{|C|}} H_{1, N}\left(\mathbf{z}_{c_{1}}\right) \ldots H_{J, N}\left(\mathbf{z}_{C_{J}}\right) d \mathbf{z}_{C}
$$

where every $c_{j}, j=1, \ldots, J$, is a subset of $C$. Note that since $|t|=t_{1}+\ldots+t_{J}>0$ in (58), there must exist $j_{1} \neq j_{2} \in\{1, \ldots, J\}$, such that $c_{0}:=c_{j_{1}} \cap c_{j_{2}} \neq \emptyset$. By the generalized Cauchy Schwartz inequality (Lemma 2.3 in [16]), one gets a bound for (161) as:

$$
\left|\int_{\mathbb{R}^{|C|}} H_{1, N}\left(\mathbf{z}_{c_{1}}\right) \ldots H_{J, N}\left(\mathbf{z}_{c_{J}}\right) d \mathbf{z}_{C}\right| \leq\left\|H_{j_{1}, N} \otimes_{\left|c_{0}\right|} H_{j_{2}, N}\right\| \prod_{j \neq j_{1}, j_{2}}\left\|H_{j, N}\right\|,
$$

where $\left\|H_{j_{1}, N} \otimes_{\left|c_{0}\right|} H_{j_{2}, N}\right\| \rightarrow 0$ as $N \rightarrow \infty$ by (59). In addition, $\left\|f_{i, j, N}\right\|, N \geq 1$ are uniformly bounded due to the tightness of the distribution of $I_{k_{i, j}}\left(f_{i, j, N}\right), N \geq 1$ (Lemma 2.1 of [16]). This, by the generalized Cauchy-Schwartz inequality (Lemma 2.3 of in [16]), implies that $\left\|h_{i, j, N}\right\|, N \geq 1$ are uniformly bounded, which further implies the uniform boundedness of $\left\|H_{j, N}\right\|, N \geq 1$. Hence (61) goes to 0 as $N \rightarrow \infty$ and thus (60) holds.

Finally, if every component of every $\mathbf{U}_{j}$ is moment-determinate, then by Theorem 3 of [20, the distribution of $\mathbf{U}:=\left(\mathbf{U}_{1}, \ldots, \mathbf{U}_{J}\right)$ is determined by its joint moments. But by (54), the joint moments of $\mathbf{U}$ are the same as if the $\mathbf{U}_{j}$ 's were independent. Then the joint moment-determinancy implies independence. 
Corollary B.2. With the notation of Theorem B.1, suppose that condition (53) is satisfied and that as $N \rightarrow \infty$, each $\mathbf{U}_{j, N}$ converges in distribution to some multivariate law which has moment-determinate components. Then there are independent random vectors $\mathbf{U}_{1}, \ldots, \mathbf{U}_{J}$ such that

$$
\left(\mathbf{U}_{1, N}, \ldots, \mathbf{U}_{J, N}\right) \stackrel{d}{\rightarrow}\left(\mathbf{U}_{1}, \ldots, \mathbf{U}_{J}\right) .
$$

Proof. Since each $\mathbf{U}_{j, N}$ converges in distribution, the vector of vectors $\left(\mathbf{U}_{1, N}, \ldots, \mathbf{U}_{J, N}\right)$ is tight in distribution, so any of its subsequence has a further subsequence converging in distribution to a vector $\left(\mathbf{U}_{1}, \ldots \mathbf{U}_{J}\right)$. But by Theorem B.1, the $\mathbf{U}_{j}$ 's are independent, and the convergence in distribution of each $\mathbf{U}_{j, N}$ implies that $\mathbf{U}_{j, N} \stackrel{d}{\rightarrow} \mathbf{U}_{j}$, and hence (62) holds.

Now we are in the position to state the result used in Theorem 3.6 in the proof of the SRD and LRD mixed case.

Theorem B.3. Consider

$$
\begin{aligned}
& \mathbf{S}_{N}=\left(I_{k_{1, S}}\left(f_{1, S, N}\right), \ldots, I_{k_{J_{S}, S}}\left(f_{J_{S}, S, N}\right)\right), \\
& \mathbf{L}_{N}=\left(I_{k_{1, L}}\left(f_{1, L, N}\right), \ldots, I_{k_{J_{L}, L}}\left(f_{J_{L}, L, N}\right)\right),
\end{aligned}
$$

where $k_{j_{S}, S}>k_{j_{L}, L}$ for all $j_{S}=1, \ldots, J_{S}$ and $j_{L}=1, \ldots, J_{L}$.

Suppose that as $N \rightarrow \infty, \mathbf{S}_{N}$ converges in distribution to a multivariate normal law, and $\mathbf{L}_{N}$ converges in distribution to a multivariate law which has moment-determinate components, then there are independent random vectors $\mathbf{Z}$ and $\mathbf{H}$, such that

$$
\left(\mathbf{S}_{N}, \mathbf{L}_{N}\right) \stackrel{d}{\rightarrow}(\mathbf{Z}, \mathbf{H}) .
$$

Proof. By Corollary B.2, we only need to check the contraction condition (53). This is done as in the proof of Theorem 4.7 of $[16$. For the convenience of the reader, we present the argument here.

Using the identity $\left\|f \otimes_{r} g\right\|^{2}=<f \otimes_{p-r} f, g \otimes_{q-r} g>$ where $r=1, \ldots, p \wedge q, f$ and $g$ have respectively $p$ and $q$ variables, we get for $r=1, \ldots, k_{i, L}$,

$$
\begin{aligned}
\left\|f_{i, S, N} \otimes_{r} f_{j, L, N}\right\|^{2} & =<f_{i, S, N} \otimes_{k_{i, S}-r} f_{j, S, N}, f_{j, L, N} \otimes_{k_{j, L}-r} f_{j, L, N}> \\
& \leq\left\|f_{i, S, N} \otimes_{k_{i, S}-r} f_{j, S, N}\right\|\left\|f_{j, L, N} \otimes_{k_{j, L}-r} f_{j, L, N}\right\| \rightarrow 0
\end{aligned}
$$

because $\left\|f_{i, S, N} \otimes_{k_{i, S}-r} f_{j, S, N}\right\| \rightarrow 0$ by the Nualart-Peccati Central Limit Theorem [17, and for the second term, one has by Cauchy-Schwartz inequality, $\left\|f_{j, L, N} \otimes_{k_{j, L}-r} f_{j, L, N}\right\| \leq\left\|f_{j, L, N}\right\|^{2}$ (generalized CauchySchwartz inequality in [16] Lemma 2.3), which is bounded due to the tightness of the distribution of $I_{k_{j, L}}\left(f_{j, L, N}\right)$ (Lemma 2.1 of [16]). Therefore (53) holds and the conclusion follows from Corollary B.2.

Acknowledgements. We would like to thank the referee for the careful reading of the paper and the comments. This work was partially supported by the NSF grant DMS-1007616 at Boston University.

\section{References}

[1] J. Beran. Statistics for Long-Memory Processes, volume 61. Chapman \& Hall/CRC, 1994.

[2] P. Billingsley. Convergence of Probability Measures. Wiley series in probability and statistics: Probability and statistics. Wiley, 1999.

[3] P. Breuer and P. Major. Central limit theorems for non-linear functionals of Gaussian fields. Journal of Multivariate Analysis, 13(3):425-441, 1983.

[4] D. Chambers and E. Slud. Central limit theorems for nonlinear functionals of stationary Gaussian processes. Probability Theory and Related Fields, 80(3):323-346, 1989. 
[5] R.L. Dobrushin and P. Major. Non-central limit theorems for non-linear functional of Gaussian fields. Probability Theory and Related Fields, 50(1):27-52, 1979.

[6] P. Doukhan, G. Oppenheim, and M.S. Taqqu. Theory and Applications of Long-Range Dependence. Birkhäuser, 2003.

[7] P. Embrechts and M. Maejima. Selfsimilar Processes. Princeton Univ Press, 2002.

[8] L. Giraitis, H.L. Koul, and D. Surgailis. Large Sample Inference for Long Memory Processes. Imperial College Press, 2012.

[9] H.C. Ho and T.C. Sun. Limiting distributions of nonlinear vector functions of stationary Gaussian processes. The Annals of Probability, pages 1159-1173, 1990.

[10] Olav Kallenberg. On an independence criterion for multiple wiener integrals. The Annals of Probability, 19(2):483-485, 1991.

[11] C. Lévy-Leduc, H. Boistard, E. Moulines, M.S. Taqqu, and V.A. Reisen. Asymptotic properties of U-processes under long-range dependence. The annals of statistics, 39(3):1399-1426, 2011.

[12] M. Lifshits. Lectures on Gaussian Processes. SpringerBriefs in Mathematics. Springer, 2012.

[13] P. Major. Multiple Wiener-Itô Integrals. Citeseer, 1981.

[14] I. Nourdin and G. Peccati. Normal Approximations With Malliavin Calculus: From Stein's Method to Universality. Cambridge Tracts in Mathematics. Cambridge University Press, 2012.

[15] I. Nourdin and J. Rosinski. Asymptotic independence of multiple Wiener-Itô integrals and the resulting limit laws. Arxiv preprint arXiv:1112.5070v1, 2011.

[16] I. Nourdin and J. Rosinski. Asymptotic independence of multiple Wiener-Itô integrals and the resulting limit laws. Arxiv preprint arXiv:1112.5070v3, 2012.

[17] D. Nualart and G. Peccati. Central limit theorems for sequences of multiple stochastic integrals. The Annals of Probability, 33(1):177-193, 2005.

[18] G. Peccati and M.S. Taqqu. Wiener Chaos: Moments, Cumulants and Diagrams: A survey with Computer Implementation. Springer Verlag, 2011.

[19] G. Peccati and C. Tudor. Gaussian limits for vector-valued multiple stochastic integrals. Séminaire de Probabilités XXXVIII, pages 219-245, 2005.

[20] LC Petersen. On the relation between the multidimensional moment problem and the one-dimensional moment problem. Mathematica Scandinavica, 51(2):361-366, 1982.

[21] V. Pipiras and M.S. Taqqu. Regularization and integral representations of Hermite processes. Statistics and probability letters, 80(23):2014-2023, 2010.

[22] Aeneas T. Rooch. Change-Point Tests for Long-Range Dependent Data. PhD thesis, University of Bochum, Germany, 2012.

[23] M.S. Taqqu. Weak convergence to fractional Brownian motion and to the Rosenblatt process. Probability Theory and Related Fields, 31(4):287-302, 1975.

[24] M.S. Taqqu. Convergence of integrated processes of arbitrary Hermite rank. Probability Theory and Related Fields, 50(1):53-83, 1979.

[25] A.S. Ustunel and M. Zakai. On independence and conditioning on Wiener space. The Annals of Probability, 17(4):1441-1453, 1989. 
Shuyang Bai and Murad S. Taqqu

Department of Mathematics and Statistics

111 Cumminton Street

Boston, MA, 02215, US

bsy9142@bu.edu

murad@bu.edu 